JOURNAL OF THE

AMERICAN MATHEMATICAL SOCIETY

Volume 8, Number 4, October 1995

\title{
LOCALIZATION FOR DERIVED CATEGORIES OF $(\mathfrak{g}, K)$-MODULES
}

\author{
JOSEPH BERNSTEIN AND VALERY LUNTS
}

\section{INTRODUCTION}

0.1. This paper arose from an attempt to solve the following problem. Let $(\mathfrak{g}, K)$ be a Harish-Chandra pair, i.e. $\mathfrak{g}$ is a complex reductive Lie algebra, and $K$ is an algebraic group with an action $K \rightarrow \operatorname{Aut}(\mathfrak{g})$ and an embedding $k=$ Lie $K \subset \mathfrak{g}$, satisfying some standard conditions (see 1.1 below). Let $Z$ be the center of the enveloping algebra $U(\mathfrak{g})$. Fix a regular character $\theta: Z \rightarrow \mathbb{C}$. Let $\mathscr{M}(\mathfrak{g}, K)$ be the category of $(\mathfrak{g}, K)$-modules and $\mathscr{M}_{\theta}(\mathfrak{g}, K) \subset \mathscr{M}(\mathfrak{g}, K)$ the subcategory consisting of modules annihilated by $\operatorname{Ker} \theta$. Then by the localization theorem this category $\mathscr{M}_{\theta}(\mathfrak{g}, K)$ can be described geometrically. Namely, fix a Borel subalgebra $\mathfrak{b} \subset \mathfrak{g}$ and a dominant weight $\lambda$ corresponding to $\theta$. Consider the algebra $D_{\lambda}$ of twisted differential operators on the flag space $X$ of $\mathfrak{g}$. Then $\mathscr{M}_{\theta}(\mathfrak{g}, K)=\mathscr{M}\left(D_{\lambda}, K\right)$, the $K$-equivariant $D_{\lambda}$-modules on $X$.

This result allows us to study many properties of Harish-Chandra modules geometrically. But it does not give a geometric interpretation of Ext-groups of modules in $\mathscr{M}_{\theta}(\mathfrak{g}, K)$. Namely, let $M, N \in \mathscr{M}_{\theta}(\mathfrak{g}, K)$. From the point of view of representation theory the interesting objects are $\operatorname{Ext}_{\mathscr{M}(\mathfrak{g}, K)}(M, N)$, the Ext-groups in the category of all $(\mathfrak{g}, K)$-modules. But these Ext's do not admit localization since arbitrary $(\mathfrak{g}, K)$-modules do not localize.

Our main result is a geometric interpretation of these Ext-groups and, more precisely, of the corresponding derived category. Let us describe it.

Let $\mathscr{M}_{\tilde{\theta}}(\mathfrak{g}, K) \subset \mathscr{M}(\mathfrak{g}, K)$ be the subcategory of $\theta$-finite modules. That is, each element $m$ of $M \in \mathscr{M}_{\tilde{\theta}}(\mathfrak{g}, K)$ is annihilated by some power of $\operatorname{Ker} \theta$. Recall the localization for the category $\mathscr{M}_{\tilde{\theta}}(\mathfrak{g}, K)$ (precise definitions will be given later). Let $G$ be the algebraic group of automorphisms of $\mathfrak{g}, H \subset G$ a maximal torus, $\mathfrak{h}=$ Lie $H$. The flag variety $X$ has a natural $H$-monodromic structure $\widetilde{X} \rightarrow X$. Let $\widetilde{\mathscr{M}}\left(D_{\widetilde{X}}\right)$ denote the category of weakly $H$-equivariant $D_{\widetilde{X}}$-modules. Elements of $\widetilde{\mathscr{M}}\left(D_{\widetilde{X}}\right)$ are called monodromic $D$-modules on $X$. The enveloping algebra $U(h)$ acts naturally by endomorphisms of $\widetilde{\mathscr{M}}\left(D_{\tilde{X}}\right)$. Let $\lambda: U(h) \rightarrow \mathbb{C}$ be a character and $\widetilde{\mathscr{K}}_{\tilde{\lambda}}\left(D_{\widetilde{X}}\right) \subset \widetilde{\mathscr{M}}\left(D_{\widetilde{X}}\right)$ be the subcategory of $\lambda$-finite modules. Suppose that $K$ fixes $\lambda$ (for example, this holds if $K$ is connected). Then we may consider the category of $K$-equivariant $\lambda$-finite monodromic modules $\widetilde{\mathscr{M}}_{\hat{\lambda}}\left(D_{\widetilde{X}}, K\right)$. Assume now that $\lambda$ is a regular dominant

Received by the editors May 18, 1994.

1991 Mathematics Subject Classification. Primary 22E47; Secondary 18E30. 
weight corresponding to the (regular) central character $\theta$. Then by the localization theorem

$$
\mathscr{M}_{\tilde{\theta}}(\mathfrak{g}, K) \simeq \widetilde{\mathscr{M}_{\tilde{\lambda}}}\left(D_{\widetilde{X}}, K\right) \text {. }
$$

Let $D^{b}(\mathfrak{g}, K)=D^{b}(\mathscr{M}(\mathfrak{g}, K))$ be the bounded derived category of $(\mathfrak{g}, K)$ modules. Consider the full subcategory $D_{\tilde{\theta}}^{b}(\mathfrak{g}, K) \subset D^{b}(\mathfrak{g}, K)$ of complexes with cohomologies in $\mathscr{M}_{\tilde{\theta}}(\mathfrak{g}, K)$. We want to find a geometric counterpart of $D_{\tilde{\theta}}^{b}(\mathfrak{g}, K)$.

Consider $\tilde{X}$ as a $K$-variety and recall the $K$-equivariant derived category

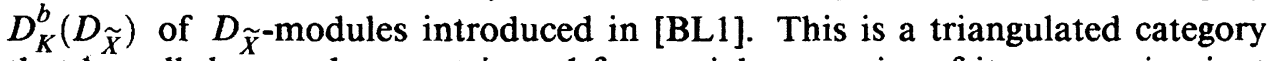
that has all the usual geometric and functorial properties of its nonequivariant analogue. Below we use the same construction as in [BL1] to define the $K$ equivariant derived category $D_{K, \text { mon }}^{b}\left(D_{\widetilde{X}}\right)$ of monodromic $D_{\widetilde{X}}$-modules. Let $D_{K, \tilde{\lambda}}^{b}\left(D_{\widetilde{X}}\right) \subset D_{K, \text { mon }}^{b}\left(D_{\tilde{X}}\right)$ be its $\lambda$-finite part. This is a certain triangulated $t$ category with the heart $\widetilde{\mathscr{A}}_{\tilde{\lambda}}\left(D_{\widetilde{X}}, K\right)$. We may apply the (equivariant) RiemannHilbert correspondence to translate the category $D_{K, \tilde{\lambda}}^{b}\left(D_{\tilde{X}}\right)$ into a certain category of $K$-equivariant constructible sheaves. In this last category the Ext-groups are computed in the category of all sheaves on a topological space, hence they have a geometric (cohomological) meaning. We prove the following

0.1.1. Theorem. Assume that $\lambda$ is a regular dominant weight corresponding to the (regular) central character $\theta$. Then there is a natural equivalence of triangulated categories

$$
D_{\tilde{\boldsymbol{\theta}}}^{b}(\mathfrak{g}, K) \simeq D_{K, \tilde{\lambda}}^{b}\left(D_{\widetilde{X}}\right) .
$$

This theorem is a natural extension to derived categories of the usual localizataion theorem. Actually, we prove a slightly more general theorem (Theorem 2.15) where we do not require $\lambda$ to be fixed by $K$.

0.2. The proof of Theorem 0.1 .1 has two essential ingredients. First of all, one proves that the central character $\theta$ in the category $D_{\tilde{\theta}}^{b}(\mathfrak{g}, K)$ can be moved inside. That is, we have the equivalence

$$
D^{b}\left(\mathscr{M}_{\tilde{\theta}}(\mathfrak{g}, K)\right) \simeq D_{\tilde{\theta}}^{b}(\mathfrak{g}, K) .
$$

This equivalence together with the usual localization provides

$$
D_{\tilde{\theta}}^{b}(\mathfrak{g}, K) \simeq D^{b}\left(\widetilde{\mathscr{A}}_{\tilde{\lambda}}\left(D_{\widetilde{X}}, K\right)\right) .
$$

The second step is to show that the category $D^{b}\left(\widetilde{\mathscr{L}}_{\hat{\lambda}}\left(D_{\widetilde{X}}, K\right)\right)$ is the "right" one, i.e. that

$$
D^{b}\left(\widetilde{\mathscr{N}}_{\tilde{\lambda}}\left(D_{\widetilde{X}}, K\right)\right) \simeq D_{K, \tilde{\lambda}}^{b}\left(D_{\widetilde{X}}\right)
$$

This last equivalence is the equivariant analogue of a well-known theorem of Beilinson [Be].

Remark. Let us notice that this equivariant analogue does not exist in general. Namely, let $Y$ be a smooth variety with an action of a group $G$. Let 
$\mathscr{M}\left(D_{Y}, G\right)$ be the abelian category of $G$-equivariant $D_{Y}$-modules, and $D_{G}^{b}\left(D_{Y}\right)$ the $G$-equivariant derived category of $D_{Y}$-modules ([BL1]). Then the derived categories $D^{b}\left(\mathscr{M}\left(D_{Y}, G\right)\right)$ and $D_{G}^{b}\left(D_{Y}\right)$ are not equivalent in general (take for example $\left.Y=p t, G=\mathbb{C}^{*}\right)$.

We prove the equivalence $(*)$ above using the intermediate category defined by Beilinson and Ginsburg. Namely, let $Y$ and $G$ be as in the last remark. One can define what we call the $h$-derived category $D_{h}^{b}\left(\mathscr{M}\left(D_{Y}, G\right)\right)$ which consists of complexes of weakly $G$-equivariant $D_{Y}$-modules equipped with some explicit homotopies. (The same construction works for monodromic modules.) By a theorem of Beilinson (unpublished) this category is always equivalent to $D_{G}^{b}\left(D_{Y}\right)$. Compared to $D_{G}^{b}\left(D_{Y}\right)$ the category $D_{h}^{b}\left(\mathscr{M}\left(D_{Y}, G\right)\right)$ has the advantage of being defined in terms of $D$-modules on the space $Y$ itself (whereas to define $D_{G}^{b}\left(D_{Y}\right)$ one has to use free resolutions $P \rightarrow Y$ of the $G$-space $Y$ ). However, the category $D_{G}^{b}\left(D_{Y}\right)$ is more geometric and in particular provides a geometric interpretation of the Ext-groups.

To complete the proof of $(*)$ we show that

$$
D^{b}\left(\widetilde{\mathscr{M}}\left(D_{\widetilde{X}}, K\right)\right) \simeq D_{h, \tilde{\lambda}}^{b}\left(\widetilde{\mathscr{H}}\left(D_{\widetilde{X}}, K\right)\right)
$$

where $D_{h, \widetilde{\lambda}}^{b}\left(\widetilde{\mathscr{M}}\left(D_{\widetilde{X}}, K\right)\right)$ is the corresponding $h$-derived category with $\lambda$-finite cohomologies.

0.3. The technical heart of the paper consists of proving several statements (in geometric and algebraic contexts) of type $(* *)$ above. Namely, we prove that under certain conditions the usual derived category is equivalent to the corresponding $h$-derived category. For this purpose we found it useful to slightly generalize the picture and to state the main result in the language of $D G$-modules over a $D G$-algebra with a group action. Namely, we introduce the notion of a Harish-Chandra $D G$-algebra $\mathscr{B}$ such that a $\mathscr{B}$-module is a generalization of a $(\mathfrak{g}, K)$-module.

The second technical statement concerns the placement of the central character. Namely, we need several results of the form

$$
D_{\tilde{\theta}}^{b}(\mathscr{M}) \simeq D^{b}\left(\mathscr{M}_{\tilde{\theta}}\right)
$$

Here again we prove one general statement in the language of Harish-Chandra $D G$-algebras and then use its various specializations.

0.4. Sections 1 and 2 are devoted to the proof of Theorem 0.1.1. In section 1 we deal with algebraic categories "before the localization". Section 2 deals with geometric categories of $D$-modules and monodromic $D$-modules "after the localization". In particular, in section 2 we recall the basic localization theory for $(\mathfrak{g}, K)$-modules following [BB], and review the definition of the equivariant derived category of monodromic $D$-modules following [BL1]. In section 3 we discuss the question of when the natural functor

$$
D^{b}\left(\mathscr{M}\left(D_{Y}, G\right)\right) \rightarrow D_{G}^{b}\left(D_{Y}\right)
$$

(see the Remark in 0.2 above) is an equivalence. We formulate a conjecture that this is an equivalence if the $G$-action on $Y$ is free at a general point of 
$Y$. Then we consider some examples related to this conjecture and prove the conjecture in some special important cases.

\section{Algebraic Setting}

1.1. ( $\mathfrak{g}, K)$-pair. Let $\mathfrak{g}$ be a complex Lie algebra. Let $K$ be a complex algebraic group with the Lie algebra $k=$ Lie $K$. Assume that there is given a homomorphism $A d: K \rightarrow \operatorname{Aut}(\mathfrak{g})$ and an inclusion of Lie algebras $j: k \hookrightarrow \mathfrak{g}$ such that

(1) $j$ is $K$-equivariant,

(2) $d A d_{g}(x)=[j(g), x], g \in k, x \in \mathfrak{g}$.

Such a data will be called a $(\mathfrak{g}, K)$-pair.

1.1.1. Let $M$ be a vector space. Let $\rho: K \rightarrow \operatorname{Aut}(M)$ be an algebraic representation of $K$ and $\alpha: \mathfrak{g} \rightarrow \operatorname{End}(M)$ be a Lie algebra representation. Assume that $\alpha$ is $K$-equivariant, i.e.

$$
\alpha\left(A d_{a}(x)\right)=\rho(a) \alpha(x) \rho(a)^{-1}, a \in K, x \in \mathfrak{g} .
$$

Then we call $M$ a weak $(\mathfrak{g}, K)$-module.

Weak $(\mathfrak{g}, K)$-modules form an abelian category $\mathscr{M}\left(\mathfrak{g}, K^{w}\right)$.

1.1.2. A weak $(\mathfrak{g}, K)$-module is called a $(\mathfrak{g}, K)$-module if

$$
\alpha(j(g))=d \rho_{g}, \quad g \in k .
$$

The $(\mathfrak{g}, K)$-modules form an abelian category $\mathscr{M}(\mathfrak{g}, K)$. Let $D^{b}(\mathfrak{g}, K)=$ $D^{b}(\mathscr{M}(\mathfrak{g}, K))$ be the bounded derived category of $\mathscr{M}(\mathfrak{g}, K)$.

1.2. $h$-derived category. Let us recall a different derived category introduced in [DV].

Let $C_{h}(\mathfrak{g}, K)$ denote the category of complexes $C^{\cdot}$ of weak $(\mathfrak{g}, K)$-modules together with a linear map $i: k \rightarrow \operatorname{Hom}^{-1}\left(C^{*}, C^{*}\right)$ such that

(i) $i$ is $K$-equivariant, i.e. $a i_{g} a^{-1}=i_{A d_{a}(g)}, a \in K, g \in k$,

(ii) $i_{g}$ is a morphism of $\mathfrak{g}$-modules,

(iii) $i_{g_{1}} i_{g_{2}}+i_{g_{2}} i_{g_{1}}=0$,

(iv) $d i_{g}+i_{g} d=d \rho_{g}-\alpha(g)$.

We will call such a complex an h-complex (a complex with "homotopies"). The condition (iv) means that $i_{g}$ is a homotopy between operators $d \rho_{g}$ and $\alpha(g)$ on $C^{\cdot}$. In particular, the cohomologies of $C^{\cdot}$ lie in $\mathscr{M}(\mathfrak{g}, K)$.

We define the operators $i_{g}$ on the shifted $h$-complex $C^{\cdot}[1]$ as minus $i_{g}$ on $C^{\circ}$.

Given two $h$-complexes $C_{1}^{\cdot}, C_{2}^{\cdot}$ we define the complex $\operatorname{Hom}^{\cdot}\left(C_{1}^{\cdot}, C_{2}^{*}\right)$ as follows

$$
\begin{aligned}
\operatorname{Hom}^{j}\left(C_{1}^{\cdot}, C_{2}^{\cdot}\right) & =\left\{\left(f_{\ell}\right) \in \prod \operatorname{Hom}_{\mathscr{M}\left(\mathfrak{g}, K^{w}\right)}\left(C_{1}^{\ell}, C_{2}^{\ell+j}\right): f_{\ell} i_{g}-(-1)^{\ell} i_{g} f_{\ell}=0\right\}, \\
d^{j}\left(f_{\ell}\right) & =d f_{\ell}-(-1)^{j} f_{\ell-1} d .
\end{aligned}
$$


Then the group $\operatorname{Hom}_{C_{h}(\mathfrak{g}, K)}\left(C_{1}^{\cdot}, C_{2}^{\cdot}\right)$ is the degree zero cycles in $\operatorname{Hom}\left(C_{1}^{\cdot}, C_{2}^{\cdot}\right)$. We define the homotopy category $\mathscr{K}_{h}(\mathfrak{g}, K)$ in the usual way by putting $\operatorname{Hom}_{\mathscr{R}_{h}(\mathfrak{g}, K)}=H^{\circ} \operatorname{Hom}^{\cdot}\left(C_{1}^{\cdot}, C_{2}^{\cdot}\right)$. This is a triangulated category as usual. Finally we define the bounded $h$-derived category $D_{h}^{b}(\mathfrak{g}, K)$ by localizing $\mathscr{K}_{h}(\mathfrak{g}, K)$ with respect to quasi-isomorphisms. This is a triangulated category in the usual way. The abelian category $\mathscr{M}(\mathfrak{g}, K)$ is naturally identified as the full subcategory of $D_{h}^{b}(\mathfrak{g}, K)$ consisting of complexes concentrated in degree zero (this follows for example from Theorem 1.3 below).

1.3. Given a complex of $(\mathfrak{g}, K)$-modules, it may be considered as an $h$-complex with the map $i=0$. Thus we have a natural functor

$$
\alpha: D^{b}(\mathfrak{g}, K) \rightarrow D_{h}^{b}(\mathfrak{g}, K)
$$

Theorem. The functor $\alpha$ is an equivalence of categories.

This theorem follows from a more general theorem (Theorem 1.6) below.

1.4. Harish-Chandra triple. Let us introduce a more general setting. Namely, we will generalize the notion of a $(\mathfrak{g}, K)$-pair and a $(\mathfrak{g}, K)$-module.

Let $A$ be an associative $\mathbb{C}$-algebra with 1 . Let $F$ be a complex algebraic group and $K \subset F$ a subgroup such that the connected component $K^{\circ}$ is a normal subgroup of $F$. Let $k=$ Lie $K$. Assume that $F$ acts on $A$ algebraically via $\sigma: F \rightarrow \operatorname{Aut}(A)$ and there is given a map of Lie algebras $j_{A}=j: k \rightarrow A$ such that

(1) $j$ is $F$-equivariant,

(2) $d \sigma_{g}(x)=[j(g), x], g \in k, x \in A$.

We call the above data an $(A, F \mid K)$-triple or a Harish-Chandra triple. In case $F=K$ we call $(A, K \mid K)=(A, K)$ a Harish-Chandra pair. This is a generalization of the notion of a $(\mathfrak{g}, K)$-pair. Indeed, take $A=U(\mathfrak{g})$, the enveloping algebra, $F=K$ and $j=$ the inclusion $k \subset U(\mathfrak{g})$. The following definitions are the obvious generalizations of 1.1.1-2 above.

1.4.1. Let $M$ be a vector space. Let $\rho: F \rightarrow \operatorname{Aut}(M)$ be an algebraic representation of $F$ and $\alpha: A \rightarrow \operatorname{Aut}(M)$ be a representation of the algebra $A$. Assume that $\alpha$ is $F$-equivariant, i.e.

$$
\alpha\left(\sigma_{a}(x)\right)=\rho(a) \alpha(x) \rho(a)^{-1}, a \in F, x \in A .
$$

Then $M$ is called a weak $(A, F \mid K)$-module.

1.4.2. A weak $(A, F \mid K)$-module is called an $(A, F \mid K)$-module if in addition $d \rho$ and $\alpha$ agree on $k$, i.e.

$$
\alpha(j(g))=d \rho_{g}, \quad g \in k .
$$

So an $(A, F \mid K)$-module is weak for $F$ and strong for $K$.

The $(A, F \mid K)$-modules form an abelian category $\mathscr{M}(A, F \mid K)$. Let $D^{b}(A, F \mid K)=D^{b}(\mathscr{M}(A, F \mid K))$ denote the bounded derived category of $\mathscr{M}(A, F \mid K)$. We denote by $\mathscr{M}(A, K)$ and $D^{b}(\mathscr{M}(A, K))$ the corresponding categories for a Harish-Chandra pair $(A, K)$. 
1.5. Let us adjust the notion of an $h$-complex (1.2) for $(A, F \mid K)$-triples. Let $C^{\cdot}$ be a complex of weak $(A, F \mid K)$-modules together with a linear map $i: k \rightarrow$ $\operatorname{Hom}^{-1}\left(C^{\cdot}, C^{\cdot}\right)$ such that

(i) $i$ is $F$-equivariant, i.e. $a i_{g} a^{-1}=i_{A d_{a}(g)}, a \in F, g \in k$,

(ii) $i_{g}$ is a morphism of $A$-modules,

(iii) $i_{g_{1}} i_{g_{2}}+i_{g_{2}} i_{g_{1}}=0$,

(iv) $d i_{g}+i_{g} d=d \rho_{g}-\alpha(j(g))$.

Again we call such a complex an $h$-complex. The cohomologies of an $h$ complex are $(A, F \mid K)$-modules. Denote by $C_{h}(A, F \mid K)$ the abelian category of $h$-complexes.

Now we proceed exactly as in 1.2 to define the bounded $h$-derived category $D_{h}^{b}(A, F \mid K)=D_{h}^{b}(\mathscr{M}(A, F \mid K))$ (denote it by $D_{h}^{b}(\mathscr{M}(A, K))$ in case $\left.F=K\right)$. This is a triangulated category with the heart $\mathscr{M}(A, F \mid K)$.

\subsection{As in 1.3 we have the obvious functor}

$$
\beta: D^{b}(A, F \mid K) \rightarrow D_{h}^{b}(A, F \mid K)
$$

Theorem. Assume that the algebra $A$ is a projective right $U(k)$-module via the map $j: k \rightarrow A$. Then the functor $\beta$ is an equivalence of categories.

Remark. Notice that Theorem 1.3 follows from this theorem. Indeed, apply the above theorem with $A=U(\mathfrak{g}), F=K$, and $j: k \hookrightarrow \mathfrak{g}$ the given inclusion. Then $U(\mathfrak{g})$ is a free $U(k)$-module by the Poincaré-Birkhoff-Witt theorem. The above theorem is a special case of our central theorem (Theorem 1.10 below).

1.7. Later we will need the notion of a graded $(A, F \mid K)$-triple. This means that $A=\bigoplus A^{i}$ is a graded algebra with the $F$-action that preserves the grading and $j(k) \subset A^{\circ}$ (so that the conditions (1), (2) of 1.4 hold). An $(A, F \mid K)$-module in this case means a graded $(A, F \mid K)$-module (that is, a graded $A$-module with the $F$-action that preserves the grading so that the conditions in 1.1.1 and 1.4.2 hold).

1.8. Lemma. Let $(A, F \mid K)$ be a Harish-Chandra triple and $M$ be a weak $(A, F \mid K)$-module. Consider the map $w: k \rightarrow \operatorname{End}(M)$ given by

$$
w(g)=d \rho_{g}-\alpha(j(g)), \quad g \in k
$$

Then

(i) $w$ is a representation of the Lie algebra $k$,

(ii) $w(g) \in$ End $_{A} M, g \in k$,

(iii) $w$ is $F$-equivariant. 
Proof of lemma. (i) Let $g_{1}, g_{2} \in k$. Then

$$
\begin{aligned}
{\left[w\left(g_{1}\right), w\left(g_{2}\right)\right]=\left[d \rho_{g_{1}}-\alpha\left(j\left(g_{1}\right)\right), d \rho_{g_{2}}-\alpha\left(j\left(g_{2}\right)\right)\right] } \\
=\left[d \rho_{g_{1}}, d \rho_{g_{2}}\right]-\left[d \rho_{g_{1}}, \alpha\left(j\left(g_{2}\right)\right)\right] \\
\quad-\left[\alpha\left(j\left(g_{1}\right)\right), d \rho_{g_{2}}\right]+\left[\alpha\left(j\left(g_{1}\right)\right), \alpha\left(j\left(g_{2}\right)\right)\right] \\
=d \rho_{\left[g_{1}, g_{2}\right]}-\alpha\left(d \sigma_{g_{1}}\left(j\left(g_{2}\right)\right)\right)-\alpha\left(-d \sigma_{g_{2}}\left(j\left(g_{1}\right)\right)\right)+\alpha\left[j\left(g_{1}\right), j\left(g_{2}\right)\right] \\
=d \rho_{\left[g_{1}, g_{2}\right]}-\alpha\left(\left[j\left(g_{1}\right), j\left(g_{2}\right)\right]\right)+\alpha\left(\left[j\left(g_{2}\right), j\left(g_{1}\right)\right]\right)+\alpha\left[j\left(g_{1}\right), j\left(g_{2}\right)\right] \\
=d \rho_{\left[g_{1}, g_{2}\right]}-\alpha\left(j\left(\left[g_{1}, g_{2}\right]\right)\right) \\
=w\left(\left[g_{1}, g_{2}\right]\right) .
\end{aligned}
$$

(ii) Let $g \in k, x \in A$. Then

$$
\begin{aligned}
{[w(g), \alpha(x)] } & =\left[d \rho_{g}-\alpha(j(g)), \alpha(x)\right] \\
& =\left[d \rho_{g}, \alpha(x)\right]-\alpha([j(g), x]) \\
& =\alpha\left(d \sigma_{g}(x)\right)-\alpha([j(g), x]) \\
& =\alpha([j(g), x])-\alpha([j(g), x])=0 .
\end{aligned}
$$

(iii) Since the maps $d \rho, j, \alpha$ are $F$-equivariant, so is $w$.

1.8.1. Corollary. Given a Harish-Chandra triple $(A, F \mid K)$ consider the HarishChandra triple $(U(k) \otimes A, F \mid K)$, where the $F$-action on $U(k) \otimes A$ is diagonal and $j: k \rightarrow U(k) \otimes A$ is given by

$$
j: g \mapsto g \otimes 1+1 \otimes j_{A}(g), g \in k
$$

Then there exists a canonical identification $\{$ weak $(A, F \mid K)$-modules $\}=$ $\{(U(k) \otimes A, F \mid K)$-modules $\}$.

Proof. Indeed, given a weak $(A, F \mid K)$-module $M$ as in 1.4.1 define the $U(k)$ module structure on $M$ via $w(g)=d \rho_{g}-\alpha\left(j_{A}(g)\right), g \in k$. By Lemma 1.8 this makes $M$ a weak $(U(k) \otimes A, F \mid K)$-module. The definition of the structure morphism $j: k \rightarrow U(k) \otimes A$ implies that $M$ is actually a $(U(k) \otimes A, F \mid K)$ module. The converse is obvious.

1.9. Harish-Chandra $D G$-algebras. We think that the appropriate context for Theorems 1.3 and 1.6 is the language of $D G$-modules over a $D G$-algebra with a group action. So let us introduce the following general setting.

1.9.1. A Harish-Chandra differential graded algebra $\mathscr{B}$ is a four-tuple $\mathscr{B}=$ $\left(B=\bigoplus B^{i}, F \mid K, d\right)$, where $(B, F \mid K)$ is a graded Harish-Chandra triple (1.7, $1.4)$ and $(B, d)$ is a $D G$-algebra, so that

(1) the differential $d$ commutes with the $F$-action,

(2) the subspace $j(k) \subset B^{\circ}$ consists of cycles, i.e. $d(j(k))=0$.

1.9.2. Let $M=\left(\bigoplus M^{i}, d_{M}\right)$ be a complex of $\mathbb{C}$-vector spaces. We say that $M$ is a (left) $D G$-module over a Harish-Chandra $D G$-algebra $\mathscr{B}=(B, F \mid K, d)$ if $M$ is a (graded) $(B, F \mid K)$-module $(1.7,1.4 .2)$ so that the $B$-module structure makes $M$ a $D G$-module over the $D G$-algebra $(B, d)$, i.e.

$$
d_{M}(b m)=d(b) m+(-1)^{\operatorname{deg}(b)} b d_{M} m, b \in B, m \in M .
$$


We will call a $D G$-module over $\mathscr{B}$ a $\mathscr{B}$-module. A morphism of $\mathscr{B}$-modules is a morphism of graded $(B, F \mid K)$-modules which commutes with the differential. Let $\mathscr{M}(\mathscr{B})$ denote the abelian category of $\mathscr{B}$-modules.

The usual construction of the homotopy category and the derived category for complexes of modules extends to $D G$-modules over a $D G$-algebra (see for example [II], [BL1]). The same construction also works for $D G$-modules over a Harish-Chandra $D G$-algebra $\mathscr{B}$. Namely, let $M$ be a $\mathscr{B}$-module. The $B$ module structure on $M[1]$ is twisted: $b \cdot m:=(-1)^{\operatorname{deg}(b)} b m$, where $b \cdot m$ is the multiplication in $M[1]$ and $b m$ is the one in $M$. Given $M, N \in \mathscr{M}(\mathscr{B})$ define the complex $\operatorname{Hom}_{\mathscr{B}}(M, N)=\operatorname{Hom}^{\cdot}(M, N)$ as follows: $\operatorname{Hom}^{j}(M, N)=$ $\operatorname{Hom}_{\mathscr{M}(B, F \mid K)}(M, N[j])$ and $d\left(f^{j}\right)=d f^{j}-(-1)^{j} f^{j} d$ for $f^{j} \in \mathrm{Hom}^{j}$. Then the group $\operatorname{Hom}_{\mathscr{M}(\mathscr{B})}(M, N)$ is the degree zero cycle in $\operatorname{Hom}^{\circ}(M, N)$. Define the homotopy category $\mathscr{K}(\mathscr{B})$ in the usual way by putting $\operatorname{Hom}_{\mathscr{K}(\mathscr{B})}(M, N)=$ $H^{0} \operatorname{Hom}^{\cdot}(M, N)$. Localizing $\mathscr{K}(\mathscr{B})$ with respect to quasi-isomorphisms we obtain the derived category $D(\mathscr{B})$ of $\mathscr{B}$-modules. This is a triangulated category in the usual way. Denote by $D^{b}(\mathscr{B}) \subset D(\mathscr{B})$ the full subcategory generated by bounded modules $M=\bigoplus M^{i}, M^{i}=0$ for $|i| \gg 0$.

Remark. In case $B=B^{\circ}$ (and hence $d=0$ ) a $\mathscr{B}$-module is just a complex of $(B, F \mid K)$-modules and hence $D^{b}(\mathscr{B})=D^{b}(B, F \mid K)$ (1.4.2).

1.9.3. Let $\mathscr{B}=(B, F \mid K, d)$ and $\mathscr{C}=(C, F \mid K, d)$ be two Harish-Chandra $D G$-algebras. A homomorphism $\varphi: \mathscr{B} \rightarrow \mathscr{C}$ is a unitary homomorphism of graded algebras $\varphi: B \rightarrow C$ which commutes with the $F$-action, with the map $j$ and with the differential $d$. Given a homomorphism $\varphi: \mathscr{B} \rightarrow \mathscr{C}$ we may consider a $\mathscr{C}$-module as a $\mathscr{B}$-module via $\varphi$. Thus we obtain restriction of scalars functors

$$
\varphi_{*}: D(\mathscr{C}) \rightarrow D(\mathscr{B}) \text { and } \varphi_{*}: D^{b}(\mathscr{C}) \rightarrow D^{b}(\mathscr{B}) .
$$

Let $\overline{\mathscr{B}}$ and $\overline{\mathscr{C}}$ be ordinary $D G$-algebras (i.e. without group action), and let $\varphi: \overline{\mathscr{B}} \rightarrow \overline{\mathscr{C}}$ be a homomorphism which is a quasi-isomorphism. Then the corresponding functor between the derived categories of $D G$-modules

$$
\varphi_{*}: D(\overline{\mathscr{C}}) \rightarrow D(\overline{\mathscr{B}})
$$

is an equivalence (see [BL1]). This is no longer true for Harish-Chandra $D G$ algebras. In Theorem 1.14 below we give a sufficient condition for the functor

$$
\varphi_{*}: D^{b}(\mathscr{C}) \rightarrow D^{b}(\mathscr{B})
$$

to be an equivalence.

1.9.4. Lemma. Let $\mathscr{C}=(C, F \mid K, d)$ be a nonpositively graded HarishChandra $D G$-algebra. Then in the category $D(\mathscr{C})$ there exist truncation functors. That is, for any $M \in D(\mathscr{C})$ there exists an exact triangle

$$
\tau_{<0} M \rightarrow M \rightarrow \tau_{\geq 0} M
$$

where $H^{i}\left(\tau_{<0} M\right)=0$ if $i \geq 0$ and $H^{i}\left(\tau_{\geq 0} M\right)=0$ if $i<0$. 
Proof of lemma. Let $M$ be a $\mathscr{C}$-module,

$$
M=\cdots M^{-1} \stackrel{d^{-1}}{\rightarrow} M^{0} \stackrel{d^{0}}{\rightarrow} M^{1} \rightarrow \cdots .
$$

Consider the subcomplex $\tau_{<0} M \subset M$ defined by

$$
\tau_{<0} M:=\bigoplus_{i<0} M^{i} \oplus \operatorname{Im} d^{-1}
$$

Since the algebra $C$ is nonpositively graded, $\tau_{<0} M$ is actually a $C$-submodule in $M$. Hence $\tau_{<0} M$ is a $\mathscr{C}$-submodule in $M$ and the desired triangle is the short exact sequence of $\mathscr{C}$-modules

$$
\tau_{<0} M \rightarrow M \rightarrow M / \tau_{<0} M:=\tau_{\geq 0} M .
$$

1.9.5. Corollary. Let $\mathscr{C}$ be a nonpositively graded Harish-Chandra DGalgebra. Then the bounded derived category $D^{b}(\mathscr{C})$ of $\mathscr{C}$-modules is generated by the modules $M$ concentrated in one degree. Also, every module $P \in D^{b}(\mathscr{C})$ is quasi-isomorphic to a bounded module.

1.9.6. Let $\mathscr{B}$ and $\mathscr{C}$ be as in 1.9.3. We define the $D G$-algebra $\mathscr{B} \otimes \mathscr{C}$ as the tensor product of algebras $B \otimes C$ with the diagonal action of $F$ and the map $j: k \rightarrow B \otimes C:$

$$
j=j_{B} \otimes 1+1 \otimes j_{C}
$$

The differential in $B \otimes C$ is the usual one:

$$
d(b \otimes c)=d b \otimes c+(-1)^{\operatorname{deg}(b)} b \otimes d c .
$$

1.9.7. $h$-construction. Consider a graded Lie algebra $\bar{k}=k^{-1} \oplus k^{0}$, where $k^{-1}=k^{0}=k$ and the commutators $\left[k^{0}, k^{0}\right],\left[k^{0}, k^{-1}\right]$ are given by $[$,$] in k$. Consider the identity map $i d=d: k^{-1} \rightarrow k^{0}$ as a derivation of $\bar{k}$ of degree 1 . The universal enveloping algebra $U(\bar{k})=\wedge^{\bullet} k \otimes U(k)$ is a nonpositively graded (by the degrees of $\wedge^{*} k$ ) associative algebra with the derivation $d$ of degree 1 induced by the above derivation of $\bar{k}$. So $U(\bar{k})$ is a $D G$-algebra.

The group $F$ acts on $U(\bar{k})$ via the adjoint representation and we have a natural map of Lie algebras $k \rightarrow U(\bar{k}), g \mapsto 1 \otimes g$. This means that $U(\bar{k})$ is a Harish-Chandra $D G$-algebra.

Notice that $U(\bar{k})$ is just the standard complex for $U(k)$,

$$
U(\bar{k})=\cdots \rightarrow \wedge^{2} k \otimes U(k) \stackrel{d}{\rightarrow} k \otimes U(k) \stackrel{d}{\rightarrow} U(k) \rightarrow 0,
$$

which is a resolution of $\mathbb{C}$.

Definition. Let $\mathscr{B}$ be a Harish-Chandra $D G$-algebra. Then the Harish-Chandra $D G$-algebra $U(\bar{k}) \otimes \mathscr{B}(1.9 .6)$ is called the $h$-construction for $\mathscr{B}$.

The augmentation map $U(\bar{k}) \rightarrow \mathbb{C}$ induces a homomorphism of HarishChandra $D G$-algebras

$$
\psi: U(\bar{k}) \otimes \mathscr{B} \rightarrow \mathscr{B}
$$

which is a quasi-isomorphism.

The following theorem is the central technical result of this paper. 
1.10. Theorem. Let $\mathscr{B}=(B, F \mid K, d)$ be a Harish-Chandra DG-algebra concentrated in degree 0 , i.e. $B=B^{0}, d=0$. Assume that $B$ is a projective right $U(k)$-module via the map $j: k \rightarrow B$. Then the quasi-isomorphism

$$
\psi: U(\bar{k}) \otimes \mathscr{B} \rightarrow \mathscr{B}
$$

from 1.9.7 induces an equivalence of categories

$$
\psi_{*}: D^{b}(\mathscr{B}) \rightarrow D^{b}(U(\bar{k}) \otimes \mathscr{B}) .
$$

1.10.1. Remark. It suffices to prove that the functor $\psi_{*}$ is fully faithful. Indeed, by Corollary 1.9 .5 both categories are generated by modules concentrated in one degree. Let $M \in D^{b}(U(\bar{k}) \otimes \mathscr{B})$ be such a module. Then $M$ is a $U(k) \otimes B$ $=(U(\bar{k}) \otimes B)^{0}$-module. Since the augmentation ideal $I \subset U(k)$ consists of boundaries in $U(\bar{k}) \otimes \mathscr{B}$, then $M$ is actually a $U(k) \otimes B / I \otimes B=B$-module. So $M$ is in the image of the functor $\psi_{*}$.

1.11. Let us explain how Theorem 1.10 implies Theorem 1.6 and hence also Theorem 1.3.

Let $(A, F \mid K)$ be a Harish-Chandra triple. Consider it as a Harish-Chandra $D G$-algebra $\mathscr{B}=\left(B=B^{0}=A, F \mid K, d=0\right)$. Then by the remark in 1.9.2 we have a natural identification

$$
D^{b}(\mathscr{B})=D^{b}(A, F \mid K) .
$$

The following claim should be compared with Corollary 1.8.1 above.

1.11.1. Claim. There are natural identifications

$$
\begin{aligned}
& C_{h}(A, F \mid K)=\mathscr{M}(U(\bar{k}) \otimes \mathscr{B}), \\
& D_{h}^{b}(A, F \mid K)=D^{b}(U(\bar{k}) \otimes \mathscr{B})
\end{aligned}
$$

so that the functor

$$
\beta: D^{b}(A, F \mid K) \rightarrow D_{h}^{b}(A, F \mid K)
$$

is identified with

$$
\psi_{*}: D^{b}(\mathscr{B}) \rightarrow D^{b}(U(\bar{k}) \otimes \mathscr{B}) .
$$

It follows from the above claim that Theorem 1.10 implies Theorem 1.6. Let us prove the claim.

Proof of claim. Let $C^{*}$ be an $h$-complex for the triple $(A, F \mid K)$. We claim that $C^{*}$ is naturally a $(U(\bar{k}) \otimes \mathscr{B})$-module. Indeed, by Corollary 1.8 .1 it is naturally a complex of $(U(k) \otimes A, F \mid K)$-modules. The property (iii) in 1.5 implies that $C^{*}$ is a graded $k^{-1}$-module via the map $i: k \rightarrow \operatorname{Hom}^{-1}\left(C^{*}, C^{*}\right)$. Properties (i), (ii) in 1.5 imply that $C^{\circ}$ is a graded $\bar{k}$-module via $w$ and $i$. So $C^{\cdot}$ is a graded weak $(U(\bar{k}) \otimes A, F \mid K)$-module. Using the definition of $j: k \rightarrow U(\bar{k}) \otimes A$ (1.9.6) we see that $C^{*}$ is actually a graded $(U(\bar{k}) \otimes A, F \mid K)$-module. Moreover, by the property (iv) in 1.5 the complex $C^{\circ}$ is a $D G$-module over the $D G$-algebra $U(\bar{k}) \otimes A$. Hence $C^{\cdot}$ is a $D G$-module over the Harish-Chandra $D G$-algebra $U(\bar{k}) \otimes \mathscr{B}$. 
The above argument actually also shows the converse. Therefore we have a natural identification:

$$
C_{h}(A, F \mid K)=\mathscr{M}(U(\bar{k}) \otimes \mathscr{B}) .
$$

This identification induces the identification of the derived categories

$$
D_{h}^{b}(A, F \mid K)=D^{b}(U(\bar{k}) \otimes \mathscr{B}),
$$

which translates the functor $\beta$ into $\psi_{*}$. This proves Claim 1.11.1.

We will deduce Theorem 1.10 from a slightly more general theorem (Theorem 1.14) below. Let us first introduce some useful constructions.

1.12. Induction and coinduction functors. Let $\mathscr{C}=(C, F \mid K, d)$ be a HarishChandra $D G$-algebra. Let $C(F$-mod $)$ be the category of complexes of algebraic $F$-modules and $\mathscr{K}(F-\bmod )$ be the corresponding homotopy category. We have the obvious forgetful functor

$$
\text { For: } \mathscr{M}(\mathscr{C}) \rightarrow C(F \text {-mod }) \text {. }
$$

Let us define left and right adjoint functors to For.

Let $M \in C(F$-mod $)$. Consider $M$ as a left $k$-module. Consider the algebra $C$ as a right $k$-module via the structure map $j: k \rightarrow C$. Consider the complex

$$
i_{\mathscr{C}}(M)=i(M):=C \otimes_{k} M
$$

as a left $C$-module with the diagonal action of $F$. This makes $i(M)$ a $D G$ module over $\mathscr{C}$. Thus we get a functor

$$
i: C(F \text {-mod }) \rightarrow \mathscr{M}(\mathscr{C})
$$

called the induction.

1.12.1. Proposition. Let $M \in C(F-\bmod )$ and $N \in \mathscr{M}(\mathscr{C})$. Then there is a natural isomorphism of complexes

$$
\operatorname{Hom}_{\mathscr{C}}(i(M), N)=\operatorname{Hom}_{F}(M, \operatorname{For}(N)) \text {. }
$$

Proof. Let $\alpha \in \operatorname{Hom}_{\mathscr{C}}(i(M), N)$. Define $\beta \in \operatorname{Hom}_{F}(M, \operatorname{For}(N))$ as follows:

$$
\beta(m):=\alpha(1 \otimes m) \text {. }
$$

One checks directly that $\beta$ is well defined and the correspondence $\alpha \mapsto \beta$ is an isomorphism of complexes.

1.12.2. Corollary. The induction functor $i_{\mathscr{C}}$ is the left adjoint to the forgetful functor For. The same is true on the level of the homotopy categories.

1.12.3. Let $M \in C(F-\bmod )$. Consider $M$ as a left $k$-module. Consider the algebra $C$ as a left $k$-module via the structure map $j: k \rightarrow C$. Put $c i_{\mathscr{C}}(M)=$ $c i(M):=\operatorname{Hom}_{k}(C, M)^{F \text {-alg }}$-the subcomplex of $\operatorname{Hom}_{k}(C, M)$ consisting of $F$-algebraic elements. We claim that $c i(M)$ is naturally a $\mathscr{C}$-module. Namely, let $f \in \operatorname{ci}(M)$. Put

$$
\begin{aligned}
d(f) & :=d f-(-1)^{\operatorname{deg}(f)} f d, \\
(a f)(c) & :=a\left(f\left(a^{-1} c\right)\right), a \in F, c \in C, \\
(c f)\left(c^{\prime}\right) & :=(-1)^{\left(\operatorname{deg}(f)+\operatorname{deg}\left(c^{\prime}\right)\right) \operatorname{deg}(c)} f\left(c^{\prime} c\right), c, c^{\prime} \in C .
\end{aligned}
$$


Thus we get a functor

$$
c i: C(F-\bmod ) \rightarrow \mathscr{M}(\mathscr{C})
$$

called the coinduction.

1.12.4. Proposition. Let $M \in C(F-\bmod )$ and $N \in \mathscr{M}(\mathscr{C})$. Then there exists a natural isomorphism of complexes

$$
\operatorname{Hom}_{\mathscr{C}}(N, c i(M))=\operatorname{Hom}_{F}(\operatorname{For}(N), M) .
$$

Proof. Let $\alpha \in \operatorname{Hom}_{\mathscr{C}}^{\cdot}(N, \operatorname{ci}(M))$. Define $\beta \in \operatorname{Hom}_{F}(\operatorname{For}(N), M)$ as follows:

$$
\beta(n):=\alpha(n)(1) \text {. }
$$

One checks directly that $\beta$ is well defined and the correspondence $\alpha \mapsto \beta$ is an isomorphism of complexes.

1.12.5. Corollary. The coinduction functor $c i$ is the right adjoint to the forgetful functor For. The same is true on the level of the homotopy categories.

1.13. Lemma. Let $\mathscr{B}$ and $\psi: U(\bar{k}) \otimes \mathscr{B} \rightarrow \mathscr{B}$ be as in Theorem 1.10 and $M \in C(F-\bmod )$. Then the induced map

$$
\psi \otimes i d: i_{U(\bar{k}) \otimes \mathscr{B}}(M) \rightarrow i_{\mathscr{B}}(M)
$$

is a quasi-isomorphism.

Proof of lemma. We may (and will) assume that $M$ is a single finite-dimensional $F$-module. Let us analyze the complex $i_{U(\bar{k}) \otimes \mathscr{B}}(M)$. Recall that the Lie algebra $k$ is mapped to $U(\bar{k}) \otimes B$ by

$$
g \mapsto g \otimes 1+1 \otimes j(g),
$$

where $j: k \rightarrow B$ is the structure map for $B$. So in $(U(\bar{k}) \otimes B) \otimes_{k} M$ we have the relation

$$
u g \otimes b \otimes m+u \otimes b j(g) \otimes m=u \otimes b \otimes g m .
$$

This means that

$$
(U(\bar{k}) \otimes B) \otimes_{k} M=U(\overline{\bar{k}}) \otimes_{k}(B \otimes M),
$$

where $B \otimes M$ is a left $k$-module via

$$
g(b \otimes m)=-b j(g) \otimes m+b \otimes g m
$$

and $U(\bar{k})$ is a right $k$-module via the right multiplication.

Recall that the complex $U(\bar{k})$ is a resolution of $\mathbb{C}$ consisting of free right $U(k)$-modules (1.9.7). Hence the lemma follows from the following.

\subsubsection{Sublemma. The left $k$-module $B \otimes M$ is projective.}

Proof of sublemma. Consider the functor

$$
P \mapsto P \otimes M
$$

from left $k$-modules to left $k$-modules. It has a left and right adjoint functor

$$
Q \mapsto Q \otimes M^{*}
$$


where $M^{*}$ is the dual vector space to $M$ ([BG], 2.1). Indeed, to a morphism $\varphi: P \rightarrow Q \otimes M^{*}$ corresponds a morphism $\varphi^{\prime}: P \otimes M \rightarrow Q$ defined by $\varphi^{\prime}(p \otimes m)=\sum\left\langle m_{i}^{*}, m\right\rangle q_{i}$, where $\varphi(p)=\sum q_{i} \otimes m_{i}^{*}$. Hence for a left $k$-module $P$ we have

$$
\operatorname{Hom}_{k}(B \otimes M, P)=\operatorname{Hom}_{k}\left(B, P \otimes M^{*}\right) .
$$

Since by our assumption $B$ is a projective left $k$-module for the action

$$
g(b):=-b j(g), g \in k, b \in B,
$$

we conclude that the $k$-module $B \otimes M$ is projective. This proves the sublemma and Lemma 1.13.

1.14. Theorem. Let $\mathscr{A}=(A, F \mid K, d)$ and $\mathscr{C}=(C, F \mid K, d)$ be nonpositively graded Harish-Chandra DG-algebras. Let $\varphi: \mathscr{A} \rightarrow \mathscr{C}$ be a quasi-isomorphism.

(i) Assume that for every injective algebraic $F$-module $T$ the induced map of complexes

$$
c i_{\mathscr{C}}(T) \rightarrow c i_{\mathscr{A}}(T)
$$

is a quasi-isomorphism. Then the functor of restriction of scalars

$$
\varphi_{*}: D^{b}(\mathscr{C}) \rightarrow D^{b}(\mathscr{A})
$$

is fully faithful.

(ii) The assumption in (i) is satisfied if the following condition holds: For every finite-dimensional $F$-module $M$ the induced map of complexes

$$
\varphi \otimes 1: i_{\mathscr{A}}(M) \rightarrow i_{\mathscr{C}}(M)
$$

is a quasi-isomorphism.

1.14.1. Remark. Theorem 1.14 implies Theorem 1.10. Indeed, put $\mathscr{C}=\mathscr{B}$, $\mathscr{A}=U(\bar{k}) \otimes \mathscr{B}, \varphi=\psi$. Then by Lemma 1.13 the condition in (ii) of Theorem 1.14 holds. Hence by part (i) of the theorem the functor

$$
\psi_{*}: D^{b}(\mathscr{B}) \rightarrow D^{b}(U(\bar{k}) \otimes \mathscr{B})
$$

is fully faithful, which suffices by Remark 1.10.1. So it suffices to prove Theorem 1.14 .

1.15. Proof of Theorem 1.14. In order to compute the group $\operatorname{Hom}_{D^{b}(\mathscr{E})}(M, N)$ for $M, N \in D^{b}(\mathscr{C})$ we need to take a $\mathscr{K}$-injective resolution $N \rightarrow I$ of $N$. Let us recall what this means.

1.15.1. Lemma-definition ([Sp]). An object $I \in D(\mathscr{C})$ is called $\mathscr{K}$-injective if it satisfies one of the following equivalent conditions

(a) $\operatorname{Hom}_{\mathscr{K}(\mathscr{E})}(P, I)=\operatorname{Hom}_{D(\mathscr{C})}(P, I)$ for all $P \in D(\mathscr{C})$

(b) $\operatorname{Hom}^{\circ}(Q, I)$ is acyclic if the module $Q \in D(\mathscr{C})$ is acyclic.

1.15.2. Example. Let $J \in C(F$ - mod ) be a bounded below complex consisting of injective algebraic $F$-modules. It follows from Corollary 1.12.5 that the coinduced $\mathscr{C}$-module $c i_{\mathscr{E}}(J)$ is $\mathscr{K}$-injective. 
1.15.3. Construction of resolution. Fix a bounded below $\mathscr{C}$-module $N=$ $\bigoplus_{j \geq \mu} N^{j}$. We will construct a special $\mathscr{K}$-injective resolution $N \rightarrow I$ of $N$.

Step 1. Choose a bounded below complex $J_{0}:=\bigoplus_{j \geq \mu} J^{j}$ of injective algebraic $F$-modules together with an $F$-morphism

$$
\theta: \operatorname{For}(N) \rightarrow J_{0},
$$

so that $\theta$ is injective and induces an injection of the cohomology groups $\theta$ : $H(N) \hookrightarrow H\left(J_{0}\right)$. Let

$$
\delta: N \rightarrow c i_{\mathscr{E}}\left(J_{0}\right)
$$

be the $\mathscr{C}$-morphism corresponding to $\theta$ by the adjunction property 1.12 .5 . Then $\delta$ has the same properties: it is injective and induces an injection on the cohomology $\delta: H(N) \hookrightarrow H\left(c i_{\mathscr{C}}\left(J_{0}\right)\right)$.

Step 2. Replace the module $N$ by the quotient $c i_{\mathscr{C}}\left(J_{0}\right) / N$ and repeat Step 1 for $c i_{\mathscr{C}}\left(J_{0}\right) / N$. Notice that since the algebra $C$ is nonpositively graded the module $c i_{\mathscr{E}}\left(J_{0}\right)$ is bounded below by the same degree $\mu$. Proceeding in this way we will get a complex

$$
0 \rightarrow N \stackrel{\delta}{\rightarrow} I_{0} \stackrel{\varepsilon_{0}}{\rightarrow} I_{1} \stackrel{\varepsilon_{1}}{\rightarrow} I_{2} \cdots
$$

of $\mathscr{C}$-modules with the following properties:

(1) Each $I_{s}$ is a coinduced module $I_{s}=c i_{\mathscr{C}}\left(J_{s}\right)$, where $J_{s}=\bigoplus_{j \geq \mu} J_{s}^{j}$ is a bounded below (by the degree $\mu$ ) complex of injective algebraic $F$ modules. Hence $I_{s}$ is also bounded below by the degree $\mu$. By 1.15 .2 the $\mathscr{C}$-module $I_{s}$ is $\mathscr{K}$-injective.

(2) The induced complex of cohomologies

$$
0 \rightarrow H(N) \rightarrow H\left(I_{0}\right) \rightarrow H\left(I_{1}\right) \rightarrow \cdots
$$

is exact.

Step 3. Define inductively the $\mathscr{C}$-modules $I_{\leq n}$ in the following way:

$$
\begin{aligned}
& I_{\leq 0}:=I_{0}, \\
& I_{\leq 1}:=C\left(\varepsilon_{0}\right)[-1]=\left(\text { cone of } I_{0} \stackrel{\varepsilon_{0}}{\rightarrow} I_{1}\right)[-1] .
\end{aligned}
$$

Notice that $\varepsilon_{1}$ defines a morphism

$$
\varepsilon_{1}^{\prime}: I_{\leq 1} \rightarrow I_{2}
$$

put

$$
I_{\leq 2}:=C\left(\varepsilon_{1}^{\prime}\right)[-1]
$$

and so on.

So as a graded $C$-module, $I_{\leq n}=I_{0} \oplus I_{1}[-1] \oplus \cdots \oplus I_{n}[-n]$. The $\mathscr{C}$-modules $I_{\leq n}$ are $\mathscr{K}$-injective and they form the obvious inverse system

$$
I_{\leq 0} \leftarrow I_{\leq 1} \leftarrow I_{\leq 2} \leftarrow \cdots ;
$$

put

$$
I:=\lim _{\leftarrow} I_{\leq n}
$$


So as a graded $C$-module

$$
I=\prod I_{s}[-s]
$$

The map $\delta: N \rightarrow I_{0}$ defines a compatible set of morphisms $\delta_{n}: N \rightarrow I_{\leq n}$, and hence a morphism $\delta: N \rightarrow I$. It follows from the property (2) in Step 2 that this morphism is a quasi-isomorphism.

Step 4. Claim. The $\mathscr{C}$-module I is $\mathscr{H}$-injective.

The proof is easy (using the construction of $I$ and property (b) in 1.15.1).

So we have constructed a $\mathscr{K}$-injective resolution $\delta: N \rightarrow I$ so that the module $I$ is bounded below by the same degree $\mu$ as $N$.

1.15.4. Corollary. Let $\mathscr{E}$ be a nonpositively graded Harish-Chandra DGalgebra. Let $P, Q \in D(\mathscr{E})$ be two $\mathscr{E}$-modules. Assume that

$$
P=\bigoplus_{s \leq a} P^{s}, Q=\bigoplus_{t \geq b} Q^{t} \text { and } a<b .
$$

Then $\operatorname{Hom}_{D(\mathscr{E})}(P, Q)=0$.

Proof. Indeed, let $Q \rightarrow I$ be a $\mathscr{K}$-injective resolution constructed in 1.15.2. Then $I=\bigoplus_{j \geq b} I^{j}$. Hence

$$
\operatorname{Hom}_{D(\mathscr{E})}(P, Q)=\operatorname{Hom}_{\mathscr{K}(\mathscr{E})}(P, I)=0
$$

1.15.5. Now we are ready to prove part (i) of Theorem 1.14. So assume that for every injective algebraic $F$-module $T$ the natural map of complexes

$$
c i_{\mathscr{C}}(T) \rightarrow c i_{\mathscr{A}}(T)
$$

is a quasi-isomorphism. Then the same is true if we replace $T$ by a bounded below complex of injective $F$-algebraic modules.

Fix $M, N \in D^{b}(\mathscr{C})$. We need to show that

$$
\operatorname{Hom}_{D(\mathscr{C})}(M, N)=\operatorname{Hom}_{D(\mathscr{A})}(M, N) .
$$

By Corollary 1.9 .5 we may (and will) assume that $N$ is bounded below, $N=\bigoplus_{j \geq \mu} N^{j}$, and $M$ is bounded above, $M=\bigoplus_{j \leq \nu} M^{j}$.

Let $N \rightarrow I$ be the $\mathscr{K}$-injective resolution of $N$ constructed in 1.15.2. Then

$$
\operatorname{Hom}_{D(\mathscr{C})}(M, N)=\operatorname{Hom}_{D(\mathscr{C})}(M, I)
$$

and

$$
\operatorname{Hom}_{D(\mathscr{A})}(M, N)=\operatorname{Hom}_{D(\mathscr{A})}(M, I) .
$$

It follows from Corollary 1.15.4 that there exists $n \gg 0$ such that

$$
\operatorname{Hom}_{D(\mathscr{C})}(M, I)=\operatorname{Hom}_{D(\mathscr{E})}\left(M, I_{\leq n}\right)
$$

and

$$
\operatorname{Hom}_{D(\mathscr{A})}(M, I)=\operatorname{Hom}_{D(\mathscr{A})}\left(M, I_{\leq n}\right)
$$

(see Step 3 in 1.15 .3 for the definition of $I_{\leq n}$ ). But $I_{\leq n}$ is a successive cone of morphisms between the modules $I_{s}=c i_{\mathscr{C}}\left(J_{s}\right)$. So it suffices to prove that

$$
\operatorname{Hom}_{D(\mathscr{C})}\left(M, c i_{\mathscr{C}}\left(J_{s}\right)\right)=\operatorname{Hom}_{D(\mathscr{A})}\left(M, c i_{\mathscr{E}}\left(J_{s}\right)\right) \text {. }
$$


By our assumption $c i_{\mathscr{C}}\left(J_{s}\right) \simeq c i_{\mathscr{A}}\left(J_{s}\right)$. Now

$\operatorname{Hom}_{D(\mathscr{C})}\left(M, c i_{\mathscr{C}}\left(J_{s}\right)\right)=\operatorname{Hom}_{\mathscr{X}(\mathscr{C})}\left(M, c i_{\mathscr{C}}\left(J_{s}\right)\right)=\operatorname{Hom}_{\mathscr{K}(F-\bmod )}\left(M, J_{s}\right)$,

$\operatorname{Hom}_{D(\mathscr{A})}\left(M, c i_{\mathscr{A}}\left(J_{s}\right)\right)=\operatorname{Hom}_{\mathscr{K}(\mathscr{A})}\left(M, c i_{\mathscr{A}}\left(J_{s}\right)\right)=\operatorname{Hom}_{\mathscr{R}(F-\bmod )}\left(M, J_{s}\right)$,

where the last equality in each row follows from Corollary 1.12.5. This proves (i) in Theorem 1.14. Let us prove part (ii).

1.15.6. Lemma. Let $P$ be a complex of algebraic $F$-modules. Denote the action of $F$ by $\rho: F \rightarrow \operatorname{Aut}(P)$. Assume in addition that $P$ has a structure of a left and right $k$-module (in an $F$-equivariant way) so that

$$
d \rho_{g}(p)=g p-p g, g \in k, p \in P .
$$

Consider the following functors from the category $C(F-\bmod )$ to itself:

$$
\begin{aligned}
& E \mapsto P_{k} \otimes_{k} E, \\
& H \mapsto \operatorname{Hom}_{k}\left({ }_{k} P,{ }_{k} H\right)^{F \text {-alg }} .
\end{aligned}
$$

The $F$-action on $P \otimes E$ and $\operatorname{Hom}^{\circ}(P, H)$ is defined as in 1.12, 1.12.3. Namely, given $a \in F$

$$
\begin{aligned}
a(p \otimes e) & :=a p \otimes a e, \quad p \in P, \quad e \in E, \\
a(f)(p) & :=a\left(f\left(a^{-1} p\right)\right), \quad p \in P, f \in \operatorname{Hom}^{\circ}(P, H) .
\end{aligned}
$$

Then there exists a natural isomorphism of complexes

$$
\operatorname{Hom}_{F}^{\cdot}\left(E, \operatorname{Hom}_{k}\left({ }_{k} P,{ }_{k} H\right)^{F-\text { alg }}\right)=\operatorname{Hom}_{F}\left(P_{k} \otimes_{k} E, H\right) .
$$

Proof. Given $\alpha \in \operatorname{Hom}_{F}^{\cdot}(P \otimes E, H)$ define $\beta \in \operatorname{Hom}_{F}^{\cdot}\left(E, \operatorname{Hom}^{\bullet}(P, H)^{F \text {-alg }}\right)$ by the formula

$$
\beta(e)(p)=(-1)^{\operatorname{deg}(e) \operatorname{deg}(p)} \alpha(p \otimes e) .
$$

One checks directly that the map $\alpha \mapsto \beta$ is well defined and is an isomorphism of complexes.

1.15.7. Corollary. The functors

$$
\begin{aligned}
& E \mapsto P_{k} \otimes_{k} E, \\
& H \mapsto \operatorname{Hom}_{k}\left({ }_{k} P,{ }_{k} H\right)^{F-\text { alg }}
\end{aligned}
$$

as in Lemma 1.15 .6 are adjoint on the level of the categories $C(F-\bmod )$ and $\mathscr{K}(F-\bmod )$.

1.15.8. We are ready to prove part (ii) of Theorem 1.14. So assume that for every finite-dimensional $F$-module $M$ the induced morphism of complexes

$$
A_{k} \otimes_{k} M \rightarrow C_{k} \otimes_{k} M
$$

is a quasi-isomorphism. Let $T$ be an injective algebraic $F$-module. We need to prove that the induced map of complexes

$$
\gamma: \operatorname{Hom}_{k}\left({ }_{k} C,{ }_{k} T\right)^{F-\text { alg }} \rightarrow \operatorname{Hom}_{k}\left({ }_{k} A,{ }_{k} T\right)^{F-\text { alg }}
$$

is a quasi-isomorphism. 
Since the algebra structure on $A$ and $C$ plays no role in these statements, we are actually working in the context of Lemma 1.15 .6 and it suffices to prove that $\gamma$ is a quasi-isomorphism of $F$-complexes.

It suffices to prove that $\gamma$ induces an isomorphism

$$
\operatorname{Hom}_{\mathscr{R}(F-\bmod )}\left(M, c i_{\mathscr{E}}(T)\right)=\operatorname{Hom}_{\mathscr{R}(F-\bmod )}\left(M, c i_{\mathscr{A}}(T)\right)
$$

for every finite-dimensional $F$-module $M$. Using Corollary 1.15.7 this is the same as proving the isomorphism

$$
\operatorname{Hom}_{\mathscr{K}(F-\bmod )}\left(i_{\mathscr{C}}(M), T\right)=\operatorname{Hom}_{\mathscr{K}(F-\bmod )}\left(i_{\mathscr{A}}(M), T\right) .
$$

But this follows from the quasi-isomorphism

$$
i_{\mathscr{A}}(M) \simeq i_{\mathscr{E}}(M)
$$

and the fact that $T$ is injective.

This proves part (ii) in Theorem 1.14. Hence we proved Theorems 1.14, $1.10,1.6,1.3$.

1.16. Introduction of the central character. Our next goal is to show how to introduce the central character in Theorems $1.3,1.6,1.10,1.14$ above. It is again convenient to work in the context of Harish-Chandra $D G$-algebras.

Let $\mathscr{B}=(B, F \mid K, d)$ be a nonpositively graded Harish-Chandra $D G$ algebra. Consider the category $\mathscr{M}(\mathscr{B})$ of $\mathscr{B}$-modules. Let $Z$ be a finitely generated $F$-invariant subring of the center of $B$ consisting of cycles, i.e. $d(Z)=0$. Let $I \subset Z$ be an $F$-invariant ideal. Let $M \in \mathscr{M}(\mathscr{B})$. An element $m \in M$ is called $I$-finite if $I^{n} m=0$ for $n \gg 0$. Put $M^{\tilde{I}}:=\bigcup_{n} \operatorname{Ker} I^{n} \subset M$ the submodule of $I$-finite elements. We say that $M$ is $I$-finite if $M^{\tilde{I}}=M$. Let $\mathscr{M}_{\tilde{I}}(\mathscr{B}) \subset \mathscr{M}(\mathscr{B})$ be the full subcategory consisting of $I$-finite modules. Let $D^{b}\left(\mathscr{M}_{\tilde{I}}(\mathscr{B})\right)$ be its bounded derived category. Consider the bounded derived category $D^{b}(\mathscr{B})$ of $\mathscr{B}$-modules. Let $D_{\tilde{I}}^{b}(\mathscr{B}) \subset D^{b}(\mathscr{B})$ be the full subcategory of modules $M$ such that the cohomology groups $H^{i}(M)$ consist of $I$-finite elements. By Corollary 1.9.5 the category $D_{\tilde{I}}^{b}(\mathscr{B})$ is generated by $\mathscr{M}_{\tilde{I}}(\mathscr{B})$. We have the obvious functor

$$
\nu: D^{b}\left(\mathscr{M}_{\tilde{I}}(\mathscr{B})\right) \rightarrow D_{\tilde{I}}^{b}(\mathscr{B}) .
$$

1.16.1. Theorem. Assume that the algebra $B$ is left Noetherian. Then the functor $\nu$ is an equivalence of categories.

1.17. Before we prove Theorem 1.16 .1 let us derive some consequences. Let us consider, for example, a $(\mathfrak{g}, K)$-pair as in 1.1. Let $Z=Z(\mathfrak{g}) \subset U(\mathfrak{g})$ be the center of the enveloping algebra and $I \subset Z$ be a $K$-invariant ideal. Let $\mathscr{M}_{I}(\mathfrak{g}, K) \subset \mathscr{M}(\mathfrak{g}, K)$ be the full subcategory consisting of $I$-finite modules and $D^{b}\left(\mathscr{M}_{\tilde{l}}(\mathfrak{g}, K)\right)$ be the corresponding derived category. Consider the full subcategory $D_{\tilde{I}}^{b}(\mathfrak{g}, K) \subset D^{b}(\mathfrak{g}, K)$ of complexes $M$ with cohomologies $H^{i}(M)$ in $\mathscr{M}_{\tilde{I}}(\mathfrak{g}, K)$. We have the natural functor

$$
\nu^{\prime}: D^{b}\left(\mathscr{M}_{\tilde{I}}(\mathfrak{g}, K)\right) \rightarrow D_{\tilde{I}}^{b}(\mathfrak{g}, K) .
$$


1.17.1 Theorem. This functor $\nu^{\prime}$ is an equivalence.

Proof. Indeed, take $B=B^{0}=U(\mathfrak{g}), F=K$ and $\mathscr{B}=(B, F \mid K, d=0)$, and apply 1.16 .1 .

We have a similar statement for the $h$-derived category $D_{h}^{b}(\mathfrak{g}, K)$. Namely, we have the similar subcategory $D_{h, \tilde{I}}^{b}(\mathfrak{g}, K) \subset D_{h}^{b}(\mathfrak{g}, K)$ and the natural functor

$$
\nu^{\prime \prime}: D_{h}^{b}\left(\mathscr{M}_{\tilde{I}}(\mathfrak{g}, K)\right) \rightarrow D_{h, \tilde{I}}^{b}(\mathfrak{g}, K) .
$$

1.17.2. Theorem. The functor $\nu^{\prime \prime}$ is an equivalence.

Proof. Indeed, put $A=U(\mathfrak{g}), F=K$ and consider the Harish-Chandra $D G$ algebra $\mathscr{B}=(U(\bar{k}) \otimes A, F \mid K, d)(1.9 .7)$. Then we have the natural identifications $(1.11 .1)$

$$
\begin{aligned}
& C_{h}(\mathfrak{g}, K)=\mathscr{M}(\mathscr{B}), \\
& D_{h}^{b}(\mathfrak{g}, K)=D^{b}(\mathscr{B}) .
\end{aligned}
$$

Hence $\nu^{\prime \prime}$ is an equivalence by 1.16.1. ing.

Combining the equivalences $\nu^{\prime}, \nu^{\prime \prime}$ with Theorem 1.3 we obtain the follow-

1.18. Theorem. There exists a natural equivalence of categories

$$
D^{b}\left(\mathscr{M}_{\tilde{I}}(\mathfrak{g}, K)\right) \rightarrow D_{h}^{b}\left(\mathscr{M}_{\tilde{I}}(\mathfrak{g}, K)\right)
$$

1.19. Remarks. 1. Theorem 1.17.1 implies that one can apply the localization to the category $D_{\tilde{I}}^{b}(\mathfrak{g}, K)$, where $I=\operatorname{Ker} \theta: Z \rightarrow \mathbb{C}$ for a regular $K$-invariant character $\theta$ of $Z=Z(U(\mathfrak{g}))$ (see 2.9 below).

2. Theorem 1.18 is a major step towards the proof of our main theorem 0.1 .1 .

3. All the results in 1.17-1.18 remain valid if we replace the $(\mathfrak{g}, K)$-pair by a Harish-Chandra triple $(A, F \mid K)$ (1.4), where the algebra $A$ is left Noetherian.

1.20. Proof of Theorem 1.16.1. Since the algebra $B$ is nonpositively graded, both categories $D^{b}\left(\mathscr{M}_{\tilde{I}}(\mathscr{B})\right)$ and $D_{\tilde{I}}^{b}(\mathscr{B})$ are generated by modules $M \in \mathscr{M}_{\tilde{I}}(\mathscr{B})$ concentrated in degree 0, i.e. $M=M^{0}(1.9 .5)$. Hence it suffices to prove that the functor $\nu$ is fully faithful.

1.20.1. Lemma. Let $N$ be a $(B, F \mid K)$-module. Then there exists $a(B, F \mid K)$ submodule $S \subset N$ such that $S \cap N^{\tilde{I}}=0$ and $N / S=(N / S)^{\tilde{I}}$.

We postpone the proof of this lemma until 1.21.2.

1.20.2. Proposition. Let $M \in D_{\tilde{I}}^{b}(\mathscr{B})$ be a bounded $\mathscr{B}$-module. Then there exists a morphism of $\mathscr{B}$-modules

$$
\delta: M \rightarrow \bar{M},
$$

such that $\bar{M} \in D^{b}\left(\mathscr{M}_{\tilde{I}}(\mathscr{B})\right)$ and $\delta$ is a quasi-isomorphism.

Proof of proposition. Let $M$ be the bounded $\mathscr{B}$-module

$$
0 \rightarrow M^{i} \stackrel{d^{i}}{\rightarrow} M^{i+1} \ldots \stackrel{d^{n-1}}{\rightarrow} M^{n} \rightarrow 0 .
$$


Let $t$ be the smallest integer such that $\left(M^{t}\right)^{\tilde{I}} \neq M^{t}$. Consider the $B$-submodule $N:=\bigoplus_{j=i}^{t} M^{j}$ of $M$. Let $S \subset N$ be as in Lemma 1.20.1. Then $S \subset M^{t}$.

Claim 1. $\operatorname{Ker} d^{t} \cap S=0$.

Indeed, let $m \in S, d m=0$. Since $H^{t}(M)$ is $I$-finite, we have $I^{p} m \subset$ $\operatorname{Im} d^{t-1}$ for $p \gg 0$. But $M^{t-1}$ is $I$-finite, hence $I^{q} m=0$ for some $q \gg 0$. Hence $m=0$.

Claim 2. $S \oplus d^{t} S$ is a $B$-submodule of $M$.

Indeed, it suffices to prove that for $b \in B^{-1}, s \in S$ we have $b \cdot d s \in S$. But $0=d(0)=d(b s)=d b \cdot s-b \cdot d s$ and $d b \cdot s \in S$.

So we constructed an acyclic $\mathscr{B}$-submodule $S \oplus d^{t}(S)$ of $M$. Replace $M$ by $\tilde{M}=M / S \oplus d^{t}(S)$ and repeat the construction for $\tilde{M}$. (Notice that $\tilde{M}^{t}=\left(\tilde{M}^{t}\right)^{\tilde{I}}$.) Finally we arrive at a $\mathscr{B}$-module $\bar{M} \in \mathscr{M}_{\tilde{I}}(\mathscr{B})$ together with a morphism

$$
\delta: M \rightarrow \bar{M}
$$

which is a quasi-isomorphism. This proves the proposition.

1.20.3. Now we can finish the proof of Theorem 1.16.1. Let $P, Q \in$ $D^{b}\left(\mathscr{M}_{\tilde{I}}(\mathscr{B})\right)$ and fix $f \in \operatorname{Hom}_{D_{l}^{b}(\mathscr{B})}(P, Q)$. Then $f$ can be represented by a diagram in $\mathscr{M}(\mathscr{B})$

$$
P \stackrel{g}{\rightarrow} T \stackrel{s}{\leftarrow} Q,
$$

where $T \in D_{\tilde{I}}^{b}(\mathscr{B})$ and $s$ is a quasi-isomorphism. Let $\delta: T \rightarrow \bar{T}$ be the quasiisomorphism as in Proposition 1.20.2 (with $M=T$ ). Then $\bar{T} \in D^{b}\left(\mathscr{M}_{\tilde{I}}(\mathscr{B})\right.$ ) and the morphism $f$ is also represented by the diagram in $\mathscr{M}_{\tilde{I}}(\mathscr{B})$

$$
P \stackrel{\delta g}{\rightarrow} \bar{T} \stackrel{\delta s}{\leftarrow} Q .
$$

Hence $f$ comes from $\operatorname{Hom}_{D^{b}\left(\mathscr{M}_{I}(\mathscr{B})\right)}(P, Q)$. The same argument proves that the map

$$
\operatorname{Hom}_{D^{b}\left(\mathscr{M}_{i}(\mathscr{B})\right)}(P, G) \rightarrow \operatorname{Hom}_{D_{I}^{b}(\mathscr{B})}(P, Q)
$$

is injective. So the functor $\nu$ is fully faithful. This proves the theorem.

1.21. Let us prove Lemma 1.20.1. The proof is based on the following lemma.

1.21.1. Lemma (Artin-Rees). Let $P$ be a finitely generated $B$-module, and $T \subset$ $P$ a submodule. Then there exists $\ell \gg 0$ such that

$$
T \cap I^{s+\ell} P=I^{s} T
$$

for all $s>0$.

The proof is standard (see for example [AM]).

1.21.2 Proof of Lemma 1.20.1. Assume that $N \neq N^{I}$ (otherwise take $S=0$ ).

Claim. There exists a nonzero $(B, F \mid K)$-submodule $Q \subset N$ such that $Q \cap$ $N^{\tilde{I}}=0$.

Indeed, choose $n \in N$ such that $I^{k} n \neq 0$ for all $k>0$. Let $P \subset N$ be the $B$-submodule generated by the $F$-span $\langle F n\rangle$ of $n$. Then $P$ is a finitely 
generated $B$-module, since $\operatorname{dim}\langle F n\rangle<\infty$, and $P$ is $F$-invariant. Let $T=$ $P^{\tilde{I}} \subset P$ be the $I$-finite submodule. Notice that the $B$-module $T$ is finitely generated since $B$ is left Noetherian. Therefore applying the Artin-Rees lemma 1.21.1 we will find $t \gg 0$ such that $I^{t} P \cap T=0$. Clearly $I^{t} P \neq 0$ and is $F$ invariant. So we can take $Q=I^{t} P \subset N$. This proves the claim.

Now let $S \subset N$ be a maximal $(B, F \mid K)$-submodule such that $S \cap N^{\tilde{I}}=0$. Notice that $S \neq 0$ by the previous claim. We claim that $N / S=(N / S)^{\tilde{I}}$. Indeed suppose not. Then by the previous claim there exists a nonzero $(B, F \mid K)$ submodule $Q \subset N / S$ such that $Q \cap(N / S)^{\tilde{I}}=0$. This contradicts the maximality of $S$. We proved Lemma 1.20.1.

\section{GeOMETRIC SETTING}

In this section "space" = "variety" = smooth algebraic variety over $\mathbb{C}$. For a morphism of varieties $f: Y \rightarrow Z$, we denote by $f^{*}, f_{*}$ the inverse and the direct image functors in the category of quasi-coherent sheaves. Our basic reference for $D$-modules is [B]. We first recall the notion of an equivariant $D$ module, a monodromic structure, the localization of $(\mathfrak{g}, K)$-modules, etc. (see [BB]).

2.1. Equivariant $D$-modules. Let $X$ be a variety and $F$ an (affine) algebraic group acting on $X$ by

$$
\mu: F \times X \rightarrow X
$$

Let $p: F \times X \rightarrow X$ be the projection map. An $F$-equivariant $\mathscr{O}_{X}$-module is a quasi-coherent $\mathscr{O}_{X}$-module $M$ together with an isomorphism

$$
\mu^{*} M \cong p^{*} M
$$

satisfying the usual cocycle condition.

The $F$-action on $X$ defines an $F$-action $\sigma$ on the sheaf $D_{X}$ of differential operators on $X$ and a Lie algebra map $j:$ Lie $F \rightarrow T_{X} \subset D_{X}$, so that

$$
d \sigma_{g}(P)=[j(g), P], \quad g \in \operatorname{Lie} F, P \in D_{X}
$$

([BB], 1.8.3).

Denote by $\mathscr{M}\left(D_{X}\right)$ the category of (left) $D_{X}$-modules. Let $M \in \mathscr{M}\left(D_{X}\right)$. We say that $M$ is weakly $F$-equivariant if $M$ is an equivariant $\mathscr{O}_{X}$-module so that the $F$-action $\rho$ on $M$ is compatible with the $D_{X}$-module structure:

$$
a(P m)=\sigma(a)(P)(a m), a \in F, P \in D_{X}, \quad m \in M .
$$

A weakly $F$-equivariant $D_{X}$-module is called an $F$-equivariant $D_{X}$-module if in addition

$$
d \rho_{g}(m)=j(g) m, g \in \text { Lie } F, m \in M .
$$

We denote the abelian categories of weakly equivariant and equivariant $D_{X^{-}}$ modules by $\mathscr{M}\left(D_{X}, F^{w}\right)$ and $\mathscr{M}\left(D_{X}, F\right)$ respectively. 
Example. Let $X=p t$. Then $\mathscr{M}\left(D_{X}, F^{w}\right)=$ \{algebraic $F$-modules and $\mathscr{M}\left(D_{X}, F\right)=\{$ representations of the group of components of $F\}$.

2.2. Monodromic varieties. Let $X$ be a variety and $H$ be an algebraic torus. An $H$-monodromic structure on $X$ is a principal homogeneous $H$-space

$$
\pi \stackrel{\tilde{X}}{\stackrel{\perp}{X}}
$$

We call a pair $(X, \tilde{X})$ an $H$-monodromic variety. A monodromic D-module on $X$ is a weakly $H$-equivariant $D_{\tilde{X}}$-module. We denote by $\tilde{\mathscr{M}}\left(D_{\tilde{X}}\right):=$ $\mathscr{M}\left(D_{\tilde{X}}, H^{w}\right)$ the category of monodromic $D$-modules.

Let $\mathfrak{h}=$ Lie $H$ and $U(\mathfrak{h})$ be the universal enveloping algebra of $\mathfrak{h}$.

Consider the following sheaf of algebras on $X$ :

$$
\tilde{D}:=\pi_{*} D_{\tilde{X}}^{H} .
$$

The $H$-action on $\tilde{X}$ identifies the Lie algebra $\mathfrak{h}$ with the vertical $H$-invariant vector fields on $\tilde{X}$. The corresponding inclusion $U(\mathfrak{h}) \subset \tilde{D}$ identifies $U(\mathfrak{h})$ with the center of $\tilde{D}$ and $\tilde{D} / \mathfrak{h} \tilde{D}=D_{X}$.

Let $\mathscr{M}(\tilde{D})$ be the category of $\tilde{D}$-modules on $X$. The categories $\tilde{\mathscr{M}}\left(D_{\tilde{X}}\right)$ and $\mathscr{M}(\tilde{D})$ are naturally equivalent. Namely, the functors

$$
\begin{gathered}
\tilde{\mathscr{M}}\left(D_{\tilde{X}}\right) \underset{\pi}{\stackrel{\pi}{\rightleftarrows}} \mathscr{M}(\tilde{D}), \\
\pi .(M):=\pi_{*} M^{H}, \pi(N):=\pi^{*} N
\end{gathered}
$$

are mutually inverse equivalences of categories. by

Let $M \in \tilde{\mathscr{M}}\left(D_{\tilde{X}}\right)$ be a monodromic $D$-module. Define an $\mathfrak{h}$-action on $M$

$$
w(g)=j(g)-d \rho_{g}, g \in \mathfrak{h} .
$$

Then as in Lemma 1.8 above we have

(i) $w$ is a representation of $\mathfrak{h}$,

(ii) $w$ commutes with the $D_{\tilde{X}}$-action,

(iii) $w$ is $H$-equivariant.

Thus monodromic $D$-modules have a natural structure of $\left(D_{\tilde{X}} \otimes U(\mathfrak{h})\right)$ modules, where $U(\mathfrak{h})$ acts via $w$. Let $I \subset U(\mathfrak{h})$ be an ideal. Given $M \in$ $\tilde{\mathscr{M}}\left(D_{\tilde{X}}\right)$ we define its $I$-finite part $M^{\tilde{I}}=\left\{m \in M \mid I^{n} m=0\right.$ for $\left.n \gg 0\right\}$. Consider the full subcategories $\tilde{\mathscr{M}}_{I}\left(D_{\tilde{X}}\right) \subset \tilde{\mathscr{M}}_{\tilde{I}}\left(D_{\tilde{X}}\right) \subset \tilde{\mathscr{M}}\left(D_{\tilde{X}}\right)$, where $\tilde{\mathscr{M}}_{I}\left(D_{\tilde{X}}\right)=$ $\{M \mid I M=0\}, \tilde{\mathscr{M}}_{\tilde{I}}\left(D_{\tilde{X}}\right)=\left\{M \mid M=M^{\tilde{I}}\right\}$. We have similar subcategories $\mathscr{M}_{I}(\tilde{D}) \subset \mathscr{M}_{\tilde{I}}(\tilde{D}) \subset \mathscr{M}(\tilde{D})$. Notice that the above functors $\pi$. $\pi^{\cdot}$ are $U(\mathfrak{h})$ linear and

$$
\pi \cdot\left(M^{\tilde{I}}\right)=\pi \cdot(M)^{\tilde{I}}
$$


Hence $\pi_{.}, \pi^{*}$ induce mutually inverse equivalences

$$
\begin{aligned}
& \tilde{\mathscr{M}}_{I}\left(D_{\tilde{X}}\right) \underset{\pi}{\stackrel{\pi}{\rightleftarrows}} \mathscr{M}_{I}(\tilde{D}), \\
& \tilde{\mathscr{M}}_{\tilde{I}}\left(D_{\tilde{X}}\right) \underset{\pi}{\stackrel{\pi}{\rightleftarrows}} \mathscr{M}_{\tilde{I}}(\tilde{D}) .
\end{aligned}
$$

We are especially interested in the case $I=\operatorname{Ker} \lambda: U(\mathfrak{h}) \rightarrow \mathbb{C}$. Denote in this case $\mathscr{M}_{I}=\mathscr{M}_{\lambda}, \mathscr{M}_{\tilde{I}}=\mathscr{M}_{\hat{\lambda}}$. Then in particular we have the equivalences

$$
\tilde{\mathscr{M}}_{\tilde{\lambda}}\left(D_{\tilde{X}}\right) \underset{\pi}{\stackrel{\pi}{\rightleftarrows}} \mathscr{M}_{\tilde{\lambda}}(\tilde{D}) \text {. }
$$

2.3. Monodromic $K$-varieties. Let $K$ be an algebraic group with a homomorphism $\tau: K \rightarrow \operatorname{Aut}(H)$. Since $\operatorname{Aut}(H)$ is discrete, $\tau$ is trivial on the connected component $K^{0}$ of $K$ and $\tau(K)$ is finite. An $H$-monodromic $K$-variety is an $H$-monodromic variety $(X, \tilde{X})$ together with a $K$-action $K \times \tilde{X} \rightarrow \tilde{X}$ such that $a h \tilde{x}=\tau(a)(h) a \tilde{x}$ for $a \in K, h \in H, \tilde{x} \in \tilde{X}$; in particular the $K$-action descends to an action $K \times X \rightarrow X$. Equivalently, this is a variety $\tilde{X}$ with an action of the $\tau$-semidirect product $K \ltimes H$ such that the $H$-action on $\tilde{X}$ is free.

Let $\tilde{\mathscr{M}}\left(D_{\tilde{X}}, K^{w}\right):=\mathscr{M}\left(D_{\tilde{X}},(K \ltimes H)^{w}\right)$ be the category of weakly $K$ - equivariant monodromic $D$-modules. Let $\tilde{\mathscr{M}}\left(D_{\tilde{X}}, K\right)$ be the category of $K$ equivariant monodromic $D$-modules. So objects of $\tilde{\mathscr{M}}\left(D_{\tilde{X}}, K\right)$ are weakly $K \times H$-equivariant $D_{\tilde{X}}$-modules, which are strongly equivariant for $K$.

We can define similar notions for $\tilde{D}$-modules on $X$. Namely, the $K$-action on $D_{\tilde{X}}$ restricts to its action $\sigma$ on $\tilde{D}=\left(\pi_{X} D_{\tilde{X}}\right)^{H}$. Moreover the image of the Lie algebra map $j: k=$ Lie $K \rightarrow T_{\tilde{X}} \subset D_{\tilde{X}}$ is contained in $H$-invariants, so we have the Lie algebra map $j: k \rightarrow \tilde{D}$, so that

$$
d \sigma_{g}(P)=[j(g), P], g \in k, P \in \tilde{D} .
$$

Let $N \in \mathscr{M}(\tilde{D})$. We say that $N$ is a weakly $K$-equivariant $\tilde{D}$-module if $N$ is an equivariant $\mathscr{O}_{X}$-module so that the $K$-action $\rho$ on $N$. is compatible with the $\tilde{D}$-module structure:

$$
a(P n)=\sigma(a)(P)(a n), a \in K, P \in \tilde{D}, n \in N .
$$

A weakly $K$-equivariant $\tilde{D}$-module is called a $K$-equivariant $\tilde{D}$-module if in addition

$$
d \rho_{g}(n)=j(g) n, \quad g \in k, n \in N .
$$

Denote by $\mathscr{M}(\tilde{D}, K)$ and $\mathscr{K}\left(\tilde{D}, K^{w}\right)$ the categories of $K$-equivariant and weakly $K$-equivariant $\tilde{D}$-modules respectively. The functors $\pi, \pi^{\circ}$ in 2.2 preserve $K$-equivariant modules and induce mutually inverse equivalences

$$
\begin{gathered}
\tilde{\mathscr{M}}\left(D_{\tilde{X}}, K^{w}\right) \underset{\pi^{*}}{\stackrel{\pi \cdot}{\rightleftarrows}} \mathscr{M}\left(\tilde{D}, K^{w}\right), \\
\tilde{\mathscr{M}}\left(D_{\tilde{X}}, K\right) \underset{\pi^{*}}{\stackrel{\pi}{\rightleftarrows}} \mathscr{M}(\tilde{D}, K) .
\end{gathered}
$$


The group $K$ acts on $U(\mathfrak{h})$ via $\tau$. For an ideal $I \subset U(\mathfrak{h})$ denote by $I^{*}:=$ $\tau(K) I$ its $K$-saturation. We have the usual full subcategories $\tilde{\mathscr{M}}_{I} \cdot\left(D_{\tilde{X}}, K\right) \subset$ $\tilde{\mathscr{M}}_{\tilde{I}}\left(D_{\tilde{X}}, K\right) \subset \tilde{\mathscr{M}}\left(D_{\tilde{X}}, K\right), \tilde{\mathscr{M}}_{i} \cdot\left(D_{\tilde{X}}, K^{w}\right) \subset \cdots$, and similarly for $\mathscr{M}(\tilde{D}, K)$ and $\mathscr{M}\left(\tilde{D}, K^{w}\right)$. The functors $\pi$., $\pi^{\cdot}$ induce mutually inverse equivalences of categories

$$
\begin{aligned}
& \tilde{\mathscr{M}}_{I^{\circ}}\left(D_{\tilde{X}}, K\right) \underset{\cdot}{\stackrel{\pi \cdot}{\rightleftarrows}} \mathscr{M}_{I^{\cdot}}(\tilde{D}, K), \\
& \tilde{\mathscr{M}}_{\tilde{I}^{*}}\left(D_{\tilde{X}}, K\right) \underset{\pi^{\pi}}{\stackrel{\pi}{\rightleftarrows}} \mathscr{M}_{\tilde{I}^{*}}(\tilde{D}, K),
\end{aligned}
$$

and similarly for weakly $K$-equivariant categories. In particular, if $I=\operatorname{Ker} \lambda$ : $U(\mathfrak{h}) \rightarrow \mathbb{C}$, denote $\mathscr{M}_{I^{\cdot}}=\mathscr{M}_{\lambda^{\cdot}}, \mathscr{M}_{\tilde{I}^{*}}=\mathscr{M}_{\tilde{\lambda}^{*}} ;$ then we obtain the equivalences

$$
\begin{gathered}
\tilde{\mathscr{M}}_{\tilde{\lambda}} \cdot\left(D_{\tilde{X}}, K\right) \underset{\pi^{*}}{\stackrel{\pi \cdot}{\rightleftarrows}} \mathscr{M}_{\tilde{\lambda}} \cdot(\tilde{D}, K), \\
\tilde{\mathscr{M}}_{\tilde{\lambda}}\left(D_{\tilde{X}}, K^{w}\right) \underset{\pi}{\stackrel{\pi}{\rightleftarrows}} \mathscr{M}_{\tilde{\lambda}}\left(\tilde{D}, K^{w}\right) .
\end{gathered}
$$

2.4. Functoriality for monodromic modules. Let $(X, \tilde{X})$ and $(Y, \tilde{Y})$ be two monodromic $K$-varieties. A morphism between these varieties is a morphism $f: \tilde{Y} \rightarrow \tilde{X}$ which commutes with the $(K \ltimes H)$-action. Later in sections 2.112.13 we will only consider the case when $f$ is smooth. In this case we have the exact functors (we use notations of 2.3)

$$
\begin{aligned}
f^{*} & : \tilde{\mathscr{M}}\left(D_{\tilde{X}}, K^{w}\right) \rightarrow \tilde{\mathscr{M}}\left(D_{\tilde{Y}}, K^{w}\right), \\
f^{*} & : \tilde{\mathscr{M}}\left(D_{\tilde{X}}, K\right) \rightarrow \tilde{\mathscr{M}}\left(D_{\tilde{Y}}, K\right), \\
f^{*} & : \tilde{\mathscr{M}}_{\tilde{I}^{*}}\left(D_{\tilde{X}}, K\right) \rightarrow \tilde{\mathscr{M}}_{\tilde{I}}\left(D_{\tilde{Y}}, K\right) .
\end{aligned}
$$

2.5. The $h$-derived category of monodromic $D$-modules. Given a monodromic $K$-variety $(X, \tilde{X})$ we define the $h$-derived category of monodromic $K$-equivariant $D$-modules by copying the definition in 1.5 for an $(A, F \mid K)$-triple: the algebra $A$ is replaced by the sheaf $D_{\tilde{X}}, K=K$, and $F=K \ltimes H$.

Namely, a complex $C^{*}$ of weakly $K$-equivariant monodromic $D$-modules is called an $h$-complex if there is given a linear map $i: k \rightarrow \operatorname{Hom}^{-1}\left(C^{*}, C^{*}\right)$ satisfying the following conditions:

(i) $i$ is $K \ltimes H$-equivariant, i.e. $a i_{g} a^{-1}=i_{A d_{a}(g)}, a \in K \times H, g \in k$,

(ii) $i_{g}$ is a morphism of $D_{\tilde{X}}$-modules,

(iii) $i_{g_{1}} i_{g_{2}}+i_{g_{2}} i_{g_{1}}=0$,

(iv) $d i_{g}+i_{g} d=d \rho_{g}-\alpha(j(g))$.

Denote by $C_{h}\left(\tilde{\mathscr{M}}\left(D_{\tilde{X}}, K\right)\right)$ the abelian category of $h$-complexes. Exactly as in 1.2 and 1.5 we define the bounded $h$-derived category $D_{h}^{b}\left(\tilde{\mathscr{M}}\left(D_{\tilde{X}}, K\right)\right)$ of $K$ equivariant monodromic $D$-modules. This is a triangulated category generated by $K$-equivariant monodromic $D$-modules. 
Let as before $I \subset U(\mathfrak{h})$ be an ideal and $I^{*}=\tau(K) I$ be its $K$-saturation. Consider the full subcategory $D_{h, \tilde{I}^{\prime}}^{b}\left(\tilde{\mathscr{M}}\left(D_{\tilde{X}}, K\right)\right) \subset D_{h}^{b}\left(\tilde{\mathscr{M}}\left(D_{\tilde{X}}, K\right)\right)$ consisting of complexes with cohomologies in $\mathscr{M}_{\tilde{I}^{\circ}}\left(D_{\tilde{X}}, K\right)(2.3)$. We have a natural functor

$$
\tilde{\nu}: D_{h}^{b}\left(\tilde{\mathscr{M}}_{\tilde{I}^{*}}\left(D_{\tilde{X}}, K\right)\right) \rightarrow D_{h, \tilde{I}^{*}}^{b}\left(\tilde{\mathscr{M}}\left(D_{\tilde{X}}, K\right)\right) \text {. }
$$

\subsubsection{Theorem. The above functor $\tilde{\nu}$ is an equivalence of categories.}

This theorem is proved in 2.16 below.

2.5.2. Copying again the definition in 1.5 (with $A=\tilde{D}, F=K$ ) we define the bounded $h$-derived category $D_{h}^{b}(\mathscr{M}(\tilde{D}, K))$ of $K$-equivariant $\tilde{D}$-modules. Similarly we define the full subcategory $D_{h, \tilde{I}^{\circ}}^{b}(\mathscr{M}(\tilde{D}, K))$. The functors $\pi_{.}, \pi^{\circ}$ in 2.3 preserve $h$-complexes and induce mutually inverse equivalences of categories

$$
\begin{gathered}
D_{h}^{b}\left(\tilde{\mathscr{M}}\left(D_{\tilde{X}}, K\right)\right) \underset{\pi}{\stackrel{\pi}{\rightleftarrows}} D_{h}^{b}(\mathscr{M}(\tilde{D}, K)), \\
D_{h}^{b}\left(\tilde{\mathscr{M}}_{\tilde{I}}\left(D_{\tilde{X}}, K\right)\right) \underset{\pi}{\stackrel{\pi}{\rightleftarrows}} D_{h}^{b}\left(\mathscr{M}_{\tilde{I}^{\tilde{r}}}(\tilde{D}, K)\right), \\
\left.D_{h, \tilde{I}}^{b}\left(\tilde{\mathscr{M}}\left(D_{\tilde{X}}, K\right)\right) \underset{\pi}{\stackrel{\pi}{\rightleftarrows}} D_{h, \tilde{I}^{b}}^{b} \cdot \mathscr{M}(\tilde{D}, K)\right) .
\end{gathered}
$$

\subsubsection{Corollary. The natural functor}

$$
\nu: D_{h}^{b}\left(\tilde{\mathscr{M}}_{\tilde{I}^{(}}(\tilde{D}, K)\right) \rightarrow D_{h, \tilde{I}^{*}}^{b}(\mathscr{M}(\tilde{D}, K))
$$

is an equivalence of categories.

Indeed, this follows from 2.5.1, 2.5.2.

2.6. The flag variety. The main example of a monodromic structure arises in the following way. Let $\mathfrak{g}$ be a semisimple Lie algebra. Denote by $G$ the algebraic group of automorphisms of $\mathfrak{g}$, so that $G^{0}$ is the adjoint group and Lie $G=\mathfrak{g}$. Let $X$ be the flag variety of $\mathfrak{g}$-the variety of Borel subalgebras of $\mathfrak{g}$. Fix $x \in X$ and let $\mathfrak{b}_{x} \subset \mathfrak{g}$ be the corresponding Borel subalgebra, $B_{x} \subset G^{0}$ the corresponding Borel subgroup, and $N_{x} \subset B_{x}$ the maximal nilpotent subgroup. Let $H:=B_{x} / N_{x}$ be the Cartan subgroup of $G$. The natural action of $G^{0}$ on $X$ is transitive with the stabilizer of $x \in X$ equal to $B_{x}$, so $X=G^{0} / B_{x}$. Put $\tilde{X}:=G^{0} / N_{x}$. Then the projection

$$
\pi \stackrel{\tilde{X}}{\perp}
$$

is an $H$-monodromic structure on $X$ (where $H$ acts on $\tilde{X}$ from the right).

2.7. The extended enveloping algebra. Let $\mathfrak{g}$ and $G$ be as in 2.6. Let $U(\mathfrak{g})$ be the enveloping algebra of $\mathfrak{g}$ and $Z=Z(\mathfrak{g}) \subset U(\mathfrak{g})$ be its center. Let $\mathfrak{h} \subset \mathfrak{g}$ be the Cartan subalgebra of $\mathfrak{g}, \Delta \subset \mathfrak{h}^{*}$ the root system, $\Delta^{+}$the set 
of positive roots, $\Sigma \subset \Delta^{+}$the set of simple roots, $W$ the Weyl group, and $\delta:=\frac{1}{2} \sum_{\alpha \in \Delta^{+}} \alpha$; for $\alpha \in \Delta$ let $h_{\alpha}$ be the corresponding co-root and $\sigma_{\alpha} \in W$ the corresponding reflection. For any Borel subalgebra $\mathfrak{b} \subset \mathfrak{g}$ and $\mathfrak{n}:=[\mathfrak{b}, \mathfrak{b}]$ we have the canonical identification $\mathfrak{h}=\mathfrak{b} / \mathfrak{n}$ invariant under $G^{0}$-conjugation, and $\Delta^{+}$are the weights of the $\mathfrak{h}$-action on $\mathfrak{g} / \mathfrak{b} \cong \mathfrak{n}^{*}$. (People often use the opposite ordering of $\Delta$; following [BB] we choose the one for which dominant weights correspond to positive line bundles on the flag space.)

We will think of $W$ as the group of affine transformations of $\mathfrak{h}^{*}$ that leave $-\delta$ fixed; this defines an action of $W$ on the algebra $S(\mathfrak{h})$. One has the HarishChandra isomorphism $\gamma: Z \stackrel{\sim}{\rightarrow} S(\mathfrak{h})^{W}$. Given an ideal $I \subset S(\mathfrak{h})$ let $\gamma^{*} I \subset Z$ denote the corresponding ideal in $Z$.

Denote by $V:=U(\mathfrak{g}) \otimes_{Z} U(\mathfrak{h})$ the extended enveloping algebra. Then $U(\mathfrak{h})$ is the center of $V$. Let $\mathscr{M}(\mathfrak{g})$ and $\mathscr{M}(V)$ be the categories of left $U(\mathfrak{g})$ and $V$-modules respectively. The embedding $U(\mathfrak{g}) \subset V$ defines the functor $\mathscr{M}(V) \rightarrow \mathscr{M}(\mathfrak{g})$. Given an ideal $I \subset U(\mathfrak{h})$ and the corresponding ideal $J:=$ $\gamma^{*} I \subset Z$ denote by $\mathscr{M}_{I}(V)$ and $\mathscr{M}_{J}(\mathfrak{g})$ the categories of $V$ - and $U(\mathfrak{g})$-modules annihilated by $I$ and $J$ respectively. Consider also the category $\mathscr{M}_{\tilde{I}}(V)=$ $\left\{M \in \mathscr{M}(V) \mid\right.$ for every $m \in M, I^{n} m=0$ for $\left.n \gg 0\right\}$ and the similar category $\mathscr{M}_{\tilde{j}}(\mathfrak{g})$.

Let $\lambda: U(\mathfrak{h}) \rightarrow \mathbb{C}$ be a character and $\theta:=\lambda \cdot \gamma: Z \rightarrow \mathbb{C}$ the corresponding central character. The embedding $U(\mathfrak{g}) \subset V$ induces an isomorphism $U(\mathfrak{g}) /(\operatorname{Ker} \theta) U(\mathfrak{g}) \stackrel{\sim}{\rightarrow} V /(\operatorname{Ker} \lambda) V$. If $\lambda$ is regular, then

$$
U(\mathfrak{g}) /(\operatorname{Ker} \theta)^{n} U(\mathfrak{g}) \stackrel{\sim}{\rightarrow} V /(\operatorname{Ker} \lambda)^{n} V
$$

for all $n>0$. Denote $\mathscr{M}_{\lambda}(V):=\mathscr{M}_{(\operatorname{Ker} \lambda) V}(V), \mathscr{M}_{\hat{\lambda}}(V):=\mathscr{M}_{(\operatorname{Ker} \lambda) V}(V)$, $\mathscr{M}_{\theta}(\mathfrak{g}):=\mathscr{M}_{(\operatorname{Ker} \theta) U(\mathfrak{g})}(\mathfrak{g}), \mathscr{M}_{\hat{\theta}}(\mathfrak{g}):=\mathscr{M}_{(\operatorname{Ker} \theta) U(\mathfrak{g})}(\mathfrak{g})$. The functor $\mathscr{M}_{\lambda}(V) \rightarrow$ $\mathscr{M}_{\theta}(\mathfrak{g})$ is an equivalence of categories. The functor $\mathscr{M}_{\hat{\lambda}}(V) \rightarrow \mathscr{M}_{\tilde{\theta}}(\mathfrak{g})$ is an equivalence if and only if $\lambda$ is regular.

2.8. Localization of $\mathfrak{g}$-modules. Let $X$ be the flag space of $\mathfrak{g}$ and $(X, \tilde{X})$ the $H$-monodromic variety as in 2.6. Let $\tilde{D}$ be the sheaf of rings on $X$ as in 2.2. Recall that $U(\mathfrak{h}) \subset \tilde{D}$ and $U(\mathfrak{h})$ is the center of $\tilde{D}$. For a character $\lambda: U(\mathfrak{h}) \rightarrow \mathbb{C}$ we have the usual categories $\mathscr{M}_{\lambda}(\tilde{D})$ and $\mathscr{M}_{\tilde{\lambda}}(\tilde{D})(2.2)$.

The left $G$-action and the right $H$-action on $\tilde{X}$ define a homomorphism

$$
\beta: U(\mathfrak{g}) \otimes U(\mathfrak{h}) \rightarrow \Gamma(X, \tilde{D}) .
$$

In fact, for $z \in Z$ we have $\beta(z \otimes 1)=\beta(1 \otimes \gamma(z))$. Hence $\beta$ induces a homomorphism

$$
\tilde{\beta}: V \rightarrow \Gamma(X, \tilde{D}) .
$$

It is known that $\tilde{\beta}$ is an isomorphism ([BB], 3.2.2). This defines a pair of adjoint functors

$$
\mathscr{M}(V) \underset{\Gamma}{\stackrel{\mathscr{L}}{\rightleftarrows}} \mathscr{M}(\tilde{D})
$$

by $\Gamma(M):=\Gamma(X, M), \mathscr{L}(N):=N \otimes_{V} \tilde{D}$. These functors are $U(\mathfrak{h})$-linear, 
hence they induce the adjoint functors

$$
\mathscr{M}_{\lambda}(V) \underset{\Gamma_{i}}{\stackrel{\mathscr{L}_{\lambda}}{\rightleftarrows}} \mathscr{M}_{\lambda}(\tilde{D}), \mathscr{M}_{\tilde{\lambda}}(V) \underset{\Gamma_{\tilde{\lambda}}}{\stackrel{\mathscr{L}_{\bar{i}}}{\rightleftarrows}} \mathscr{M}_{\tilde{\lambda}}(\tilde{D}) .
$$

Recall that a weight $\lambda \in \mathfrak{h}^{*}$ is dominant if $(\lambda+\delta)\left(h_{\alpha}\right) \notin\{-1,-2, \ldots\}$ for any positive co-root $h_{\alpha} \in \mathfrak{h}$. The basic result is the following

2.8.1. Theorem ([BB], 3.3.1). If $\lambda$ is a regular dominant weight, then $\left(\Gamma_{\lambda}, \mathscr{L}_{\lambda}\right)$ are mutually inverse equivalences of categories. The functors $\left(\Gamma_{\tilde{\lambda}}, \mathscr{L}_{\bar{\lambda}}\right)$ are also equivalences of categories.

2.8.2. Remark. Combined with the equivalences $\mathscr{M}_{\lambda}(V) \cong \mathscr{M}_{\theta}(\mathfrak{g}), \mathscr{M}_{\tilde{\lambda}}(V) \cong$ $\mathscr{M}_{\tilde{\theta}}(\mathfrak{g})$ in 2.7 this theorem provides the localization for the categories $\mathscr{M}_{\theta}(\mathfrak{g})$, $\mathscr{M}_{\tilde{\theta}}(\mathfrak{g})$.

2.9. Localization for $(\mathfrak{g}, K)$-modules. Assume now that the Lie algebra $\mathfrak{g}$ is part of a $(\mathfrak{g}, K)$-pair (1.1). Then the action of $K$ on $\mathfrak{g}$ induces a $K$-action on the Cartan subalgebra $\mathfrak{h}$ (call this action $\tau$ ). Hence the monodromic variety $(X, \tilde{X})(2.6,2.8)$ is a monodromic $K$-variety. Let $\lambda: U(\mathfrak{h}) \rightarrow \mathbb{C}$ be a weight and $\theta=\lambda \cdot \gamma: Z \rightarrow \mathbb{C}$ the corresponding central character. Put $\lambda^{\cdot}:=\tau(K) \lambda \in \tau(K) \backslash \mathfrak{h}^{*}$ and $\theta^{\cdot}:=\tau(K) \theta$. We have the corresponding full sub-

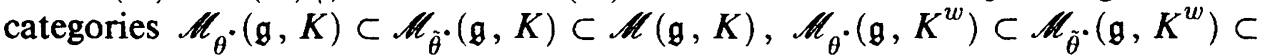
$\mathscr{M}\left(\mathfrak{g}, K^{w}\right)$ of $(\mathfrak{g}, K)$ - and weak $(\mathfrak{g}, K)$-modules. There are similar categories

$$
\begin{gathered}
\mathscr{M}_{\lambda} \cdot(V, K) \subset \mathscr{M}_{\dot{\lambda}}(V, K) \subset \mathscr{M}(V, K), \\
\mathscr{M}_{\lambda} \cdot\left(V, K^{w}\right) \subset \mathscr{M}_{\dot{\lambda}}\left(V, K^{w}\right) \subset \mathscr{M}\left(V, K^{w}\right) .
\end{gathered}
$$

As above we have the obvious functors $\mathscr{M}(V, K) \rightarrow \mathscr{M}(\mathrm{g}, K), \mathscr{M}\left(V, K^{w}\right) \rightarrow$ $\mathscr{M}\left(\mathfrak{g}, K^{w}\right)$, which induce the equivalences

$$
\mathscr{M}_{\lambda} \cdot(V, K) \stackrel{\sim}{\rightarrow} \mathscr{M}_{\theta} \cdot(\mathfrak{g}, K), \mathscr{M}_{\lambda} \cdot\left(V, K^{w}\right) \stackrel{\sim}{\rightarrow} \mathscr{M}_{\theta} \cdot\left(\mathfrak{g}, K^{w}\right) .
$$

If $\lambda$ is regular and the stabilizers of $\lambda$ and $\theta$ in $\tau(K)$ coincide (e.g. if $\tau(K) \lambda=$ $\lambda)$, then

$$
\mathscr{M}_{\tilde{\lambda}^{\prime}}(V, K) \stackrel{\sim}{\rightarrow} \mathscr{M}_{\tilde{\theta}^{*}}(\mathfrak{g}, K), \quad \mathscr{M}_{\tilde{\lambda}}\left(V, K^{w}\right) \stackrel{\sim}{\rightarrow} \mathscr{M}_{\tilde{\theta}} \cdot\left(\mathfrak{g}, K^{w}\right)
$$

are also equivalences of categories.

The functors $\Gamma$ and $\mathscr{L}$ preserve $K$-equivariant modules:

$$
\mathscr{M}(V, K) \underset{\Gamma}{\stackrel{\mathscr{L}}{\rightleftarrows}} \mathscr{M}(\tilde{D}, K)
$$

and induce the functors between the full subcategories

$$
\begin{aligned}
& \mathscr{M}_{\lambda} \cdot(V, K) \underset{\Gamma_{i}}{\stackrel{\mathscr{L}^{\cdot}}{\rightleftarrows}} \mathscr{M}_{\lambda^{*}}(\tilde{D}, K), \\
& \mathscr{M}_{\dot{\lambda}^{\prime}} \cdot(V, K) \underset{\Gamma_{\dot{\lambda}}}{\stackrel{\mathscr{L}_{\dot{\lambda}}}{\rightleftarrows}} \mathscr{M}_{\tilde{\lambda}^{\cdot}}(\tilde{D}, K) .
\end{aligned}
$$


2.9.1. Corollary ([BB], 3.3.3). If $\lambda$ is a regular dominant weight, then $\left(\Gamma_{\lambda^{\cdot}}, \mathscr{L}_{\dot{\lambda}^{*}}\right),\left(\Gamma_{\tilde{\lambda}^{*}}, \mathscr{L}_{\tilde{\lambda}^{*}}\right)$ are equivalences of categories. The same is true for weakly $K$-equivariant categories.

2.9.2. Remark. Combined with the equivalences $\mathscr{M}_{\lambda} \cdot(V, K) \stackrel{\sim}{\rightarrow} \mathscr{M}_{\theta} \cdot(\mathfrak{g}, K)$, $\mathscr{M}_{\tilde{\lambda}^{\cdot}}(V, K) \stackrel{\sim}{\rightarrow} \mathscr{M}_{\tilde{\theta}} \cdot(\mathfrak{g}, K)$ above this corollary provides the localization for the categories $\mathscr{M}_{\theta} \cdot(\mathfrak{g}, K)$ and $\mathscr{M}_{\tilde{\theta}}(\mathfrak{g}, K)$. Similarly, for the weakly $K$-equivariant categories $\mathscr{M}_{\theta} \cdot\left(\mathfrak{g}, K^{w}\right)$ and $\mathscr{M}_{\tilde{\theta}^{*}}\left(\mathfrak{g}, K^{w}\right)$.

2.10. Summarizing the results. We will use the notations of sections $2.6-2.9$ above. Fix a dominant regular weight $\lambda$. Let $\theta$ be the corresponding central character. Assume that the stabilizers of $\lambda$ and $\theta$ in $\tau(K)$ coincide (e.g. that $\tau(K) \lambda=\lambda)$. Let as before $\lambda^{\circ}=\tau(K) \lambda \in \tau(K) \backslash \mathfrak{h}^{*}$ and $\theta^{\circ}=\tau(K) \theta$ be the corresponding $K$-saturations. As was mentioned in the introduction our main goal is to provide a localization for the catetgory $D_{\tilde{\theta}^{*}}^{b}(\mathfrak{g}, K)$. Let us summarize the results we have obtained so far. Theorem 1.3 implies the equivalence of categories

$$
D_{\tilde{\theta}^{b}}^{b}(\mathfrak{g}, K) \cong D_{h, \tilde{\theta}^{\cdot}}^{b}(\mathscr{M}(\mathfrak{g}, K)) .
$$

By Theorem 1.18

$$
D_{h, \tilde{\theta}^{*}}^{b}(\mathscr{M}(\mathfrak{g}, K)) \cong D_{h}^{b}\left(\mathscr{M}_{\left.\tilde{\theta}^{(}\right)}(\mathfrak{g}, K)\right) .
$$

By Corollary 2.9.1 and Remark 2.9.2

$$
D_{h}^{b}\left(\mathscr{M}_{\tilde{\theta}^{*}}(\mathfrak{g}, K)\right) \cong D_{h}^{b}\left(\mathscr{M}_{\tilde{\lambda}} \cdot(\tilde{D}, K)\right) \text {. }
$$

By Corollary 2.5.3

$$
D_{h}^{b}\left(\mathscr{M}_{\tilde{\lambda}^{\prime}} \cdot(\tilde{D}, K)\right) \cong D_{h, \tilde{\lambda}}^{b} \cdot(\mathscr{M}(\tilde{D}, K))
$$

By 2.5.2

$$
D_{h, \tilde{\lambda}^{\circ}}^{b}(\mathscr{M}(\tilde{D}, K)) \cong D_{h, \tilde{\lambda}^{\prime}}^{b}\left(\tilde{\mathscr{M}}\left(\tilde{D}_{\tilde{X}}, K\right)\right) .
$$

So we have the natural equivalence

$$
D_{\tilde{\theta}^{*}}^{b}(\mathfrak{g}, K) \cong D_{h, \tilde{\lambda}^{\circ}}^{b}\left(\tilde{\mathscr{M}}\left(\tilde{D}_{\tilde{X}}, K\right)\right) .
$$

Thus to prove the main Theorem 0.1.1 it remains to interpret the $h$-derived category of $K$-equivariant monodromic $D$-modules $D_{h}^{b}\left(\tilde{\mathscr{M}}\left(\tilde{D}_{\tilde{X}}, K\right)\right)$ as the $K$ equivariant derived category of monodromic $D$-modules $D_{K, \text { mon }}^{b}\left(D_{\tilde{X}}\right)$ (see the definition below in 2.12).

2.11. The equivariant derived category of $D$-modules ([BL1]). Recall that "variety" = "smooth variety". Let $Y$ be a variety acted upon by an affine algebraic group $G$. Let us recall the definition of the $G$-equivariant derived category $D_{G}^{b}\left(D_{Y}\right)$ of $D_{Y}$-modules following [BL1]. In 2.12 we will extend this definition to monodromic $D$-modules. Recall the main simple principle of [BL1]: if $G$ acts freely on $Y$, then put $D_{G}^{b}\left(D_{Y}\right):=D^{b}\left(D_{G \backslash Y}\right)$; otherwise replace $Y$ by its free $G$-resolution $P \rightarrow Y$ (i.e. the $G$-action on $P$ is free) and then use $D^{b}\left(D_{G \backslash P}\right)$ as an approximation to $D_{G}^{b}\left(D_{Y}\right)$. 
A $G$-space $P$ is called free if the quotient map

$$
q \frac{P}{P}
$$

is a principal $G$-bundle. Let $P$ be a free $G$-space and $p: P \rightarrow Y$ be an affine $G$-map which is a locally trivial fibration. Then we call $P$ (or rather the pair $(P, p))$ a smooth resolution of $Y$. Smooth resolutions of $Y$ form a category $S \operatorname{Res}(Y, G)=S \operatorname{Res}(Y)$ in the obvious way. For a morphism $f: Q \rightarrow P$ of smooth resolutions denote by $\bar{f}: \bar{Q} \rightarrow \bar{P}$ the corresponding map of quotients.

2.11.1. Definition of $D_{G}^{b}\left(D_{Y}\right)$. An object $M \in D_{G}^{b}\left(D_{Y}\right)$ is a collection of objects $M_{P} \in D^{b}\left(D_{\bar{P}}\right)$ for every $P \in \operatorname{SRes}(Y)$ together with the isomorphism $\alpha_{f}$ : $\bar{f}^{*} M_{P} \stackrel{\sim}{\rightarrow} M_{Q}$ for every morphism $f: Q \rightarrow P$ in $S \operatorname{Res}(Y)$ so that given a composition of morphisms $R \stackrel{g}{\rightarrow} Q \stackrel{f}{\rightarrow} P$ we have $\alpha_{g} \cdot \bar{g}^{*} \alpha_{f}=\alpha_{f g}$. A morphism $M \rightarrow N$ in $D_{G}^{b}\left(D_{Y}\right)$ is a collection of morphisms $M_{P} \rightarrow N_{P}$ compatible with the structure isomorphisms $\alpha_{f}$.

Notice that for the "trivial" resolution $G \times Y \rightarrow Y$ we have $\overline{G \times Y}=Y$. So an object $M \in D_{G}^{b}\left(D_{Y}\right)$ in particular determines an object $M_{G \times Y} \in D^{b}\left(D_{Y}\right)$.

The abelian category $\mathscr{M}\left(D_{Y}, G\right)$ of $G$-equivariant $D_{Y}$-modules is naturally identified as a full subcategory of $D_{G}^{b}\left(D_{Y}\right)$ consisting of complexes concentrated in degree 0.

Let us recall some definitions in the spirit of [BL1]. A connected affine variety $W$ is called $n$-acyclic if $H_{D R}^{i}(W)=0,0<i \leq n$. A map of varieties $f: T \rightarrow S$ is called $n$-acyclic if $f$ is an affine map and is a locally trivial fibration with an $n$-acyclic fibre $W$. A smooth resolution $p: P \rightarrow Y$ is called $n$-acyclic if $p$ is an $n$-acyclic map. Let $J \subset \mathbb{Z}$ be a segment. For a variety $Z$ let $D^{J}\left(D_{Z}\right) \subset D^{b}\left(D_{Z}\right)$ be the full subcategory consisting of complexes, which are acyclic outside the interval $J$. Let $D_{G}^{J}\left(D_{Y}\right) \subset D_{G}^{b}\left(D_{Y}\right)$ be the full subcategory consisting of objects $M$ such that $M_{P} \in D^{J}\left(D_{\bar{P}}\right)$ for every $P \in \operatorname{SRes}(Y)$.

The main point which makes the above definition useful (and equivalent to many others) is the following.

2.11.2. Proposition. Let $P \rightarrow Y$ be an $n$-acyclic resolution and $J \subset \mathbb{Z}$ be an interval such that $|J| \leq n$. Then the functor

is fully faithful.

$$
D_{G}^{J}\left(D_{Y}\right) \rightarrow D^{J}\left(D_{\bar{P}}\right), M \mapsto M_{P}
$$

It is easy to derive the above proposition from the following.

2.11.3. Lemma. Let $f: T \rightarrow S$ be an $n$-acyclic map. Let $J \subset \mathbb{Z}$ be an interval such that $|J|<n$. Then the inverse image functor $f^{*}: D^{J}\left(D_{S}\right) \rightarrow D^{J}\left(D_{T}\right)$ is fully faithful.

Proof of lemma. Let $W$ be the fibre of $f$. Let $M, N$ be $D$-modules on $S$. It suffices to prove that

$$
f^{*}: \operatorname{Ext}_{D_{S}}^{i}(M, N) \rightarrow \operatorname{Ext}_{D_{T}}^{i}\left(f^{*} M, f^{*} N\right)
$$


is an isomorphism for $i \leq n$. Replacing $N$ by its Čech resolution we may assume that $T=S \times W$ and $f$ is the projection. But then

$$
\mathrm{Ext}_{D_{T}}^{i}\left(f^{*} M, f^{*} N\right)=\bigoplus H_{D R}^{j}(W) \otimes \mathrm{Ext}_{D_{S}}^{i-j}(M, N) .
$$

Since $H_{D R}^{0}(W)=\mathbb{C}$ and $H_{D R}^{j}(W)=0,0<j \leq n$, we get the result.

2.11.4. Remark. The $h$-derived category $D_{h}^{b}\left(D_{Y}, G\right)(2.5)$ has the advantage of being defined in terms of equivariant $D$-modules on the space $Y$ itself. However, it is easy to show that the equivariant derived category $D_{G}^{b}\left(D_{Y}\right)$ has all the usual functional properties of the nonequivariant derived category. Also by the above Proposition 2.11.2 the Ext groups in $D_{G}^{b}\left(D_{Y}\right)$ can be computed in the category of all $D$-modules on a certain space, which provides a geometric meaning for these groups and makes them computable in many cases. Fortunately the two categories $D_{h}^{b}\left(D_{Y}, G\right)$ and $D_{G}^{b}\left(D_{Y}\right)$ are equivalent (see Theorem 2.13 below).

2.12. The $K$-equivariant derived category of monodromic $D$-modules. Let us extend the above Definition 2.11 .1 to the monodromic setting. Let $(X, \tilde{X})$ be an $H$-monodromic $K$-variety (2.3). Consider $X$ as a $K$-variety and let $S \operatorname{Res}(X, K)$ be its category of smooth resolutions (2.11). For a resolution $p: P \rightarrow X$ in $S \operatorname{Res}(X, K)$ let $(P, \tilde{P})$ be the induced $H$-monodromic $K$ variety. Let $\tilde{p}: \tilde{P} \rightarrow \tilde{X}$ be the induced map. Then $\tilde{P}$ is a free $(K \ltimes H)$-space and the actions of $K^{0}$ and $H$ commute. Put $P^{0}:=K^{0} \backslash P, \tilde{P}^{0}:=K^{0} \backslash \tilde{P}, \bar{K}=$ $K / K^{0}$. Then the pair $\left(P^{0}, \tilde{P}^{0}\right)$ is an $H$-monodromic $\bar{K}$-variety, and we may consider the category $\tilde{\mathscr{M}}\left(D_{\tilde{P}^{0}}, \bar{K}\right)$ of $\bar{K}$-equivariant monodromic $D$-modules (2.3). Given a morphism $f: Q \rightarrow P$ in $\operatorname{SRes}(X, K)$ we have the induced morphism $\tilde{f}^{0}: \tilde{Q}^{0} \rightarrow \tilde{P}^{0}$ of monodromic $\bar{K}$-varieties and the inverse image functor

$$
\tilde{f}^{0 *}: D^{b}\left(\tilde{\mathscr{M}}\left(D_{\tilde{P}^{0}}, \bar{K}\right)\right) \rightarrow D^{b}\left(\tilde{\mathscr{M}}\left(D_{\tilde{Q}^{0}}, \bar{K}\right)\right)
$$

2.12.1. Definition of $D_{K, \text { mon }}^{b}\left(D_{\tilde{X}}\right)$. An object $M$ in $D_{K, \text { mon }}^{b}\left(D_{\tilde{X}}\right)$ is a collection of objects $M_{\tilde{P}} \in D^{b}\left(\tilde{\mathscr{M}}\left(D_{\tilde{P}^{0}}, \bar{K}\right)\right)$ for every $P \in S \operatorname{Res}(X, K)$ together with the isomorphism $\alpha_{f}: \tilde{f}^{0 *} M_{\tilde{P}} \stackrel{\sim}{\rightarrow} M_{\tilde{Q}}$ for every morphism $f: Q \rightarrow P$ in $\operatorname{SRes}(X, K)$ so that given a composition of morphisms $R \stackrel{g}{\rightarrow} Q \stackrel{f}{\rightarrow} P$ we have $\alpha_{g} \cdot \tilde{g}^{0 *} \alpha_{f}=\alpha_{f g}$.

2.12.2. Remark. Definition 2.12.1 reduces to 2.11 .1 in case $H=\{e\}$ and $\tilde{X}=$ $X$. Indeed, in this case $\tilde{P}^{0}=P^{0}$, which is a free $\bar{K}$-space. So $\mathscr{M}\left(D_{P^{0}}, \bar{K}\right)=$ $\mathscr{M}\left(D_{\bar{P}}\right)$ and $D^{b}\left(\mathscr{M}\left(D_{P^{0}}, \bar{K}\right)\right)=D^{b}\left(\mathscr{M}\left(D_{\bar{P}}\right)\right)$, where $\bar{P}=K \backslash P=\bar{K} \backslash P^{0}$.

The abelian cateogry $\tilde{\mathscr{M}}\left(D_{\tilde{X}}, K\right)$ of $K$-equivariant monodromic $D$-modules is naturally identified as a full subcategory of $D_{K, \text { mon }}^{b}\left(D_{\tilde{X}}\right)$ consisting of complexes concentrated in degree 0 . 
2.13. Theorem (Beilinson). Let $(X, \tilde{X})$ be an $H$-monodromic $K$-variety. Then there exists an equivalence of categories

$$
\varepsilon: D_{h}^{b}\left(\tilde{\mathscr{M}}\left(D_{\tilde{X}}, K\right)\right) \rightarrow D_{K, \text { mon }}^{b}\left(D_{\tilde{X}}\right) .
$$

Proof. The proof consists of several steps.

Step 1. Let $p: P \rightarrow X$ be a smooth resolution. Denote by $q: \tilde{P} \rightarrow \tilde{P}^{0}$ the quotient map by $K^{0}$. Since $\tilde{P}$ is a free $\left(K^{0} \times H\right)$-space the inverse image functor $q^{*}: \tilde{\mathscr{M}}\left(D_{\tilde{P}^{0}}, \bar{K}\right) \stackrel{\sim}{\rightarrow} \tilde{\mathscr{M}}\left(D_{\tilde{P}}, K\right)$ is an equivalence of categories. (The inverse functor is $\left(q^{*}\right)^{-1}=q_{*}(\cdot)^{K^{0}}$.) Hence we have the equivalence

$$
q^{*}: D^{b}\left(\tilde{\mathscr{M}}\left(D_{\tilde{P}^{0}}, \bar{K}\right)\right) \stackrel{\sim}{\rightarrow} D^{b}\left(\tilde{\mathscr{M}}\left(D_{\tilde{P}}, K\right)\right) \text {. }
$$

Step 2. We have the obvious functor

$$
\alpha: D^{b}\left(\tilde{\mathscr{M}}\left(D_{\tilde{P}}, K\right)\right) \rightarrow D_{h}^{b}\left(\tilde{\mathscr{M}}\left(D_{\tilde{P}}, K\right)\right)
$$

(see 2.5 for the definition of $D_{h}^{b}\left(\tilde{\mathscr{M}}\left(\tilde{D}_{\tilde{P}}, K\right)\right)$ ).

Claim. This is an equivalence of categories.

Proof of claim. Both categories are generated by $K$-equivariant monodromic $D$-modules $\tilde{\mathscr{M}}\left(D_{\tilde{P}}, K\right)$, so it suffices to prove that for $M, N \in \tilde{\mathscr{M}}\left(D_{\tilde{P}}, K\right)$

$$
\alpha: \operatorname{Ext}^{i}(M, N) \rightarrow \operatorname{Ext}^{i}(\alpha(M), \alpha(N))
$$

is an isomorphism. Since $\tilde{P}$ is a free $(K \ltimes H)$-space, we can find a covering $\mathscr{U}=\left\{U_{i}\right\}$ of $\tilde{P}$ by open $(K \ltimes H)$-invariant subsets of the form $U_{i}=(K \ltimes H) \times Z_{i}$ for some affine $Z_{i}$ 's. Replacing $N$ by its Čech resolution with respect to this covering we may assume that $\tilde{P}$ is an affine free $(K \ltimes H)$-space $\tilde{P}=(K \ltimes H) \times Z$. But then our claim reduces to the corresponding statement in section 1 above. Namely, put $F=K \ltimes H, A=\Gamma\left(\tilde{P}, D_{\tilde{P}}\right)$. Then $(A, F \mid K)$ is a Harish-Chandra triple (1.4) and $\tilde{\mathscr{M}}\left(D_{\tilde{P}}, K\right)=\mathscr{M}(A, F \mid K), D^{b}\left(\tilde{\mathscr{M}}\left(D_{\tilde{P}}, K\right)\right)=D^{b}(A, F \mid K)$, $D_{h}^{b}\left(\tilde{\mathscr{M}}\left(D_{\tilde{P}}, K\right)\right)=D_{h}^{b}(A, F \mid K)$. Moreover $A$ is a free $U(k)$-module $(k=$ Lie $K$ ), and so our claim follows from Theorem 1.6.

Step 3. Let us define the functor $\varepsilon$ in the theorem. Given a free resolution $p: P \rightarrow X$ we have the inverse image functor

$$
\tilde{p}^{*}: D_{h}^{b}\left(\tilde{\mathscr{M}}\left(D_{\tilde{X}}, K\right)\right) \rightarrow D_{h}^{b}\left(\tilde{\mathscr{M}}\left(D_{\tilde{P}}, K\right)\right)
$$

Compose it with the equivalences $\alpha^{-1}$ and $\left(q^{*}\right)^{-1}$ of Steps 2 and 1 to get the functor

$$
\left(q^{*}\right)^{-1} \cdot \alpha^{-1} \cdot \tilde{p}^{*}: D_{h}^{b}\left(\tilde{\mathscr{M}}\left(D_{\tilde{X}}, K\right)\right) \rightarrow D^{b}\left(\tilde{\mathscr{M}}\left(D_{\tilde{P}^{0}}, \bar{K}\right)\right) .
$$

Thus, given $M \in D_{h}^{b}\left(\tilde{\mathscr{M}}\left(D_{\tilde{X}}, K\right)\right)$, we get a compatible collection of objects $\left\{\left(q^{*}\right)^{-1} \cdot \alpha^{-1} \cdot \tilde{p}^{*}(M)\right\}_{P \in S R e s(X, K)}$, which defines the functor

$$
\varepsilon: D_{h}^{b}\left(\tilde{\mathscr{M}}\left(D_{\tilde{X}}, K\right)\right) \rightarrow D_{K, \text { mon }}^{b}\left(D_{\tilde{X}}\right) .
$$

Since both categories are generated by the $K$-equivariant monodromic $D$ modules $\tilde{\mathscr{M}}\left(D_{\tilde{X}}, K\right)$, it suffices to prove that $\varepsilon$ is fully faithful.

Step 4. In view of Proposition 2.11.2 it suffices to prove the following. 
2.13.1. Proposition. Let $p: P \rightarrow X$ be an $n$-acyclic resolution and $J \subset \mathbb{Z}$ be an interval such that $|J| \leq n$. Then the inverse image functor

$$
\tilde{p}^{*}: D_{h}^{J}\left(\tilde{\mathscr{M}}\left(D_{\tilde{X}}, K\right)\right) \rightarrow D_{h}^{J}\left(\tilde{\mathscr{M}}\left(D_{\tilde{P}}, K\right)\right)
$$

is fully faithful.

Proof of proposition. We will need some preliminary constructions.

Let $Z$ be a (smooth) variety acted upon by an affine algebraic group $F$. Consider the forgetful functor

$$
\text { For }_{w}: \mathscr{M}\left(D_{Z}, F^{w}\right) \rightarrow \mathscr{M}\left(D_{Z}\right)
$$

from weakly $F$-equivariant $D_{Z}$-modules to $D_{Z}$-modules.

2.13.2. Lemma. The functor For $_{w}$ has an exact right adjoint functor

$$
\operatorname{Ind}_{w}: \mathscr{M}\left(D_{Z}\right) \rightarrow \mathscr{M}\left(D_{Z}, F^{w}\right) .
$$

Proof of lemma. Let $\mu: F \times Z \rightarrow Z$ be the $F$-action morphism and $p_{Z}$ : $F \times Z \rightarrow Z, p_{F}: F \times Z \rightarrow F$ be the two projections. Let $M \in \mathscr{M}\left(D_{Z}\right)$. Consider $F \times Z$ as an $F$-space via the action on the first factor. Then $p_{Z}^{*} M$ is an $F$-equivariant $D_{F \times Z}$-module. Since the action map $\mu$ is $F$-equivariant, the direct image $\mu_{*} p_{Z}^{*} M$ is an $F$-equivariant $\mathscr{O}_{Z}$-module. We claim that in fact $\mu_{*} p_{Z}^{*} M$ is naturally a (weakly $F$-equivariant) $D_{Z}$-module. Indeed, let $T_{Z}$ be the tangent bundle and $\xi \in T_{Z}$. Let $\bar{\xi} \in T_{F \times Z}$ be such that $d \mu(\bar{\xi})=$ $\xi, d p_{F}(\bar{\xi})=0$. Then define the action of $\xi$ on $\mu_{*} p_{Z}^{*} M$ as the action of $\bar{\xi}$ on $p_{Z}^{*} M$. This makes $\mu_{*} p_{Z}^{*} M$ a weakly $F$-equivariant $D_{Z}$-module which we denote by $\operatorname{Ind}_{w}(M)$.

Let $i: Z \rightarrow F \times Z$ be the embedding $i(z)=(e, z)$, where $e \in F$ is the identity. We have the canonical surjection

$$
\alpha: \mu_{*} p_{Z}^{*} M \rightarrow i^{*} p_{Z}^{*} M=M
$$

which defines a morphism of functors

$$
\alpha: \text { For }_{w} \cdot \text { Ind }_{w} \rightarrow \text { Id. }
$$

Vice versa, given $N \in \mathscr{M}\left(D_{Z}, F^{w}\right)$, consider the corresponding structure map

$$
\mu^{*} N \rightarrow p_{Z}^{*} N
$$

and define

as the composition

$$
\beta(N): N \rightarrow \operatorname{Ind}_{w} \cdot \operatorname{For}_{w}(N)
$$

$$
\beta: N \hookrightarrow \mu_{*} \mu^{*} N \rightarrow \mu_{*} p_{Z}^{*} N .
$$

The morphisms $(\alpha, \beta)$ are the adjunction morphisms for the functors $\left(\right.$ For $_{w}$, Ind $_{w}$ ).

2.13.3. Corollary. The functor Ind $_{w}$ carries injective $D_{Z}$-modules to injective weakly $F$-equivariant $D_{Z}$-modules.

Let now $(Y, \tilde{Y})$ be an $H$-monodromic $K$-variety and consider the category $C_{h}\left(\tilde{\mathscr{M}}\left(D_{\tilde{Y}}, K\right)\right)$ of $h$-complexes as in 2.5 . Let $C\left(\tilde{\mathscr{M}}\left(D_{\tilde{Y}}, K^{w}\right)\right)$ be the category 
of complexes of weakly $K$-equivariant monodromic modules. Consider the natural forgetful functor

$$
\text { For }_{h}: C_{h}\left(\tilde{\mathscr{M}}\left(D_{\tilde{Y}}, K\right)\right) \rightarrow C\left(\tilde{\mathscr{M}}\left(D_{\tilde{Y}}, K^{w}\right)\right)
$$

(forgetting the "homotopies").

Let $M \in \tilde{\mathscr{M}}\left(D_{\tilde{Y}}, K^{w}\right)$ and $k=$ Lie $K$. As in lemma 1.8 define $w: k \rightarrow$ $\operatorname{End}(M)$ by

$$
w(g)=d \rho_{g}-j(g), \quad g \in k
$$

(we use the notations of 2.1). Exactly as in Lemma 1.8 one checks that

(i) $w$ is a representation of the Lie algebra $k$,

(ii) $w(g) \in$ End $_{D_{\tilde{Y}}}(M), g \in k$,

(iii) $w$ is $(K \ltimes H)$-equivariant.

Let $U(\bar{k})$ be the $D G$-algebra as in 1.9.7. Consider $U(\bar{k})$ as a left $U(k)$ module. Let us define an exact functor

$$
\operatorname{Ind}_{h}: C\left(\tilde{\mathscr{M}}\left(D_{\tilde{Y}}, K^{w}\right)\right) \rightarrow C_{h}\left(\tilde{\mathscr{M}}\left(D_{\tilde{Y}}, K\right)\right)
$$

as follows. Given a complex $C \in C\left(\tilde{\mathscr{M}}\left(D_{\tilde{Y}}, K^{w}\right)\right)$, consider it as a left $U(k)$ module via $w$. Then put

$$
\operatorname{Ind}_{h}(C):=\operatorname{Hom}_{U(k)}^{\cdot}(U(\bar{k}), C) .
$$

2.13.4. Lemma. Given $C \in C\left(\tilde{\mathscr{M}}\left(D_{\tilde{Y}}, K^{w}\right)\right)$ and $C^{\prime} \in C_{h}\left(\tilde{\mathscr{M}}\left(D_{\tilde{Y}}, K\right)\right)$ there is a canonical isomorphism of complexes

$$
\operatorname{Hom}^{\circ}\left(\operatorname{For}_{h}\left(C^{\prime}\right), C\right) \simeq \operatorname{Hom}^{\circ}\left(C^{\prime}, \operatorname{Ind}_{h}(C)\right) \text {. }
$$

In particular Ind $_{h}$ is the right adjoint to For $_{h}$ both on the level of complexes and homotopy categories.

2.13.5. Corollary. The functor $\mathrm{Ind}_{h}$ preserves $\mathscr{K}$-injectives.

2.13.6. End of the proof of Proposition 2.13.1. Consider again the monodromic $K$-variety $(X, \tilde{X})$. Let $C\left(\mathscr{M}\left(D_{\tilde{X}}\right)\right)$ be the abelian category of complexes of $D_{\tilde{X}}$-modules. Summarizing the results of $2.13 .2,2.13 .3$ (with $Z=\tilde{X}, F=$ $K \ltimes H)$ and $2.13 .4,2.13 .5$, we obtain an exact functor

$$
\operatorname{Ind}_{h} \cdot \operatorname{Ind}_{w}: C\left(\mathscr{M}\left(D_{\tilde{X}}\right)\right) \rightarrow C_{h}\left(\tilde{\mathscr{M}}\left(D_{\tilde{X}}, K\right)\right)
$$

which is the right adjoint to the forgetful functor For $_{w} \cdot$ For $_{h}$ and preserves $\mathscr{K}$-injectives.

Given $N \in D_{h}^{b}\left(\tilde{\mathscr{M}}\left(D_{\tilde{X}}, K\right)\right)$ we can construct (as in 1.15.3) a right resolution

$$
0 \rightarrow N \rightarrow I_{0} \rightarrow I_{1} \rightarrow \cdots,
$$

where $I_{j}=\operatorname{Ind}_{h} \cdot \operatorname{Ind}_{w}\left(C_{j}\right)$ for some $\mathscr{K}$-injective $C_{j} \in C\left(\mathscr{M}\left(D_{\tilde{X}}\right)\right)$.

Fix $M, N \in D_{h}^{J}\left(\tilde{\mathscr{M}}\left(D_{\tilde{X}}, K\right)\right)$. We need to show that

$$
\tilde{p}^{*}: \operatorname{Hom}(M, N) \rightarrow \operatorname{Hom}\left(\tilde{p}^{*}(M), \tilde{p}^{*}(N)\right)
$$

is an isomorphism. Replacing $N$ by the above resolution $N \rightarrow I$ we see that it suffices to show that

$$
\tilde{p}^{*}: D^{J}\left(D_{\tilde{X}}\right) \rightarrow D^{J}\left(D_{\tilde{P}}\right)
$$


is an equivalence, which is true by Lemma 2.11.3. This proves Proposition 2.13.1 and Theorem 2.13.

2.14. Let again $(X, \tilde{X})$ be an $H$-monodromic $K$-variety, $U(\mathfrak{h})$ the enveloping algebra of $\mathfrak{h}=$ Lie $H, I \in U(\mathfrak{h})$ an ideal, and $I^{\cdot}=\tau(K) I$ its $K$-saturation. Consider the full subcategory $D_{K, \tilde{I}^{\circ}}^{b}\left(D_{\tilde{X}}\right) \subset D_{K, \text { mon }}^{b}\left(D_{\tilde{X}}\right)$ consisting of complexes with cohomologies in $\tilde{\mathscr{M}}_{\tilde{I}^{*}}\left(D_{\tilde{X}}, K\right)$ (see 2.3). Then the equivalence $\varepsilon$ in Theorem 2.13 induces an equivalence

$$
\varepsilon: D_{h, \tilde{I}^{*}}^{b}\left(\tilde{\mathscr{M}}\left(D_{\tilde{X}}, K\right)\right) \stackrel{\sim}{\rightarrow} D_{K, \tilde{I}}^{b}\left(D_{\tilde{X}}\right) .
$$

If $I=\operatorname{Ker} \lambda: U(\mathfrak{h}) \rightarrow \mathbb{C}$ is a character, put $D_{K, \tilde{\lambda}^{\cdot}}^{b}\left(D_{\tilde{X}}\right):=D_{K, I^{*}}^{b}\left(D_{\tilde{X}}\right)$.

Combining the last equivalence $\varepsilon$ with the results in 2.10 we obtain the following main theorem.

2.15. Theorem. Let $(\mathfrak{g}, K)$ be a Harish-Chandra pair (1.1), where $\mathfrak{g}$ is a semisimple Lie algebra. Let $\mathfrak{h} \subset \mathfrak{g}$ be the Cartan subalgebra, $Z$ the center of $U(\mathfrak{g})$, and $\theta: Z \rightarrow \mathbb{C}$ a regular central character. Let $\lambda: U(\mathfrak{h}) \rightarrow \mathbb{C}$ be a regular dominant weight corresponding to $\theta$. Assume that the stabilizers in $K$ of $\lambda$ and $\theta$ coincide $(e . g$. $K \lambda=\lambda)$. Let $X=$ flag variety for $\mathfrak{g}$ and $(X, \tilde{X})$ be the corresponding monodromic variety. Then there exists a natural equivalence of categories

$$
D_{\tilde{\theta}^{*}}^{b}(\mathfrak{g}, K) \rightarrow D_{K, \tilde{\lambda}^{*}}^{b} \cdot\left(D_{\tilde{X}}\right)
$$

2.16. Introduction of the central character. Let us fill in the gap and prove Theorem 2.5.1 above. The idea of the proof is the exact geometric analogue of the corresponding algebraic argument. Namely, we first show that the $h$-derived category $D_{h}^{b}\left(\mathscr{M}_{\tilde{X}}, K\right)$ of $K$-equivariant monodromic $D$-modules is the same as the derived category of $D G$-modules over a certain (geometric) Harish-Chandra $D G$-algebra (example 3 in 2.16.2). Then we prove a general theorem analogous to 1.16.1 above for such Harish-Chandra $D G$-algebras.

2.16.1. Harish-Chandra $D G$-algebras (geometric case). Let $Y$ be a smooth variety with an action of an affine algebraic group $F$. Let $K \subset F$ be a subgroup such that $K^{0}$ is a normal subgroup of $F$. Put $k=$ Lie $K$.

Let $B_{Y}=\bigoplus B_{Y}^{i}$ be a sheaf of unitary graded algebras on $Y$ with a ring homomorphism $\mathscr{O}_{Y} \rightarrow B_{Y}^{0}$ which makes $B_{Y}$ a quasicoherent left $\mathscr{O}_{Y}$-module. Suppose that $B_{Y}$ is given a structure of an $F$-equivariant $\mathscr{O}_{Y}$-module $(2.1)$ so that the $F$-action preserves the ring structure on $B_{Y}$. Denote this $F$-action by $\sigma$. Let $j: U(k) \rightarrow \Gamma\left(Y, B_{Y}^{0}\right)$ be an $F$-equivariant ring homomorphism such that

$$
d \sigma_{g}(b)=[j(g), b], g \in k, b \in B_{Y} .
$$

Suppose in addition that there is given a differential $d$ on $B_{Y}$ of degree one, which makes $B_{Y}$ a sheaf of $D G$-algebras. Assume that $d$ commutes with the $F$-action and that $j(k) \subset \Gamma\left(Y, B_{Y}^{0}\right)$ consists of cycles, i.e. $d(j(k))=0$. We denote the above data $\mathscr{B}_{Y}:=\left(B_{Y}, F \mid K, d\right)$ and call it a Harish-Chandra $D G$ algebra. Obviously, this is a direct generalization of the same notion when $Y=p t$ (1.9.1). 
Let $\mathscr{B}_{Y}$ be a Harish-Chandra $D G$-algebra. Let $\left(M=\bigoplus M^{i}, d_{M}\right)$ be a complex of $F$-equivariant quasicoherent $\mathscr{O}_{Y}$-modules. Denote the $F$-action on $M$ by $\rho$. Assume that $M$ has a structure of a sheaf of (left) $D G$-modules over the sheaf of $D G$-algebras $B_{Y}$. Denote the multiplication map $\alpha: B_{Y} \rightarrow$ $\operatorname{End}(M)$. We say that $M$ is a $\mathscr{B}_{Y}$-module if

$$
\alpha\left(\sigma_{a}(b)\right)=\rho(a) \alpha(b) \rho(a)^{-1}, a \in F, b \in B_{Y},
$$

and

$$
\alpha(j(g))=d \rho_{g}, g \in k .
$$

Let $\mathscr{M}\left(\mathscr{B}_{Y}\right)$ denote the abelian category of $\mathscr{B}_{Y}$-modules. Exactly as in 1.9.2 we define the derived category $D\left(\mathscr{B}_{Y}\right)$ and its full subcategory $D^{b}\left(\mathscr{B}_{Y}\right) \subset$ $D\left(\mathscr{B}_{Y}\right)$.

2.16.2. Examples. Let $(X, \tilde{X})$ be a monodromic $K$-variety (2.3) and $F=$ $K \ltimes H$ be the $\tau$-semidirect product. Let us describe some Harish-Chandra $D G$-algebras on $\tilde{X}$.

1. Consider the differential operators $D_{\tilde{X}}$ as a graded algebra concentrated in degree zero. It has a natural $F$-action and a homomorphism $j: k \rightarrow$ $\Gamma\left(\tilde{X}, D_{\tilde{X}}\right)(2.1)$ satisfying the above conditions. So $\mathscr{D}_{\tilde{X}}:=\left(D_{\tilde{X}}, F \mid K, d=0\right)$ is a Harish-Chandra $D G$-algebra.

2. Consider the sheaf of algebras $U(k) \otimes D_{\tilde{X}}$ with the diagonal $F$-action and the structure morphism $j_{w}: k \rightarrow \Gamma\left(\tilde{X}, U(k) \otimes D_{\tilde{X}}\right)$ given by

$$
j_{w}(g)=g \otimes 1+1 \otimes j(g) .
$$

Then $U(k) \otimes \mathscr{D}_{\tilde{X}}:=\left(U(k) \otimes D_{\tilde{X}}, \quad F \mid K, \quad d=0\right)$ is a Harish-Chandra $D G$ algebra. As in 1.8.1 (remark 1.9.2) we have a natural identification

$$
D^{b}\left(U(k) \otimes \mathscr{D}_{\tilde{X}}\right)=D^{b}\left(\tilde{\mathscr{M}}\left(D_{\tilde{X}}, K^{w}\right)\right)
$$

(see 2.3).

3. Let $U(\bar{k})$ be the $D G$-algebra as in 1.9.7. Consider $U(\bar{k}) \otimes D_{\tilde{X}}$ with the diagonal $F$-action and the structure morphism $j_{h}: k \rightarrow \Gamma\left(\tilde{X}, U(\bar{k}) \otimes D_{\tilde{X}}\right)$ given by

$$
j_{h}(g)=g \otimes 1+1 \otimes j(g) .
$$

Then $U(\bar{k}) \otimes \mathscr{D}_{\tilde{X}}:=\left(U(\bar{k}) \otimes D_{\tilde{X}}, F \mid K, d\right)$ is a nonpositively graded HarishChandra $D G$-algebra. As in 1.11.1 we have natural identifications (2.5)

$$
\begin{aligned}
& \mathscr{M}\left(U(\bar{k}) \otimes \mathscr{D}_{\tilde{X}}\right)=C_{h}\left(\tilde{\mathscr{M}}\left(D_{\tilde{X}}, K\right)\right), \\
& D^{b}\left(U(\bar{k}) \otimes \mathscr{D}_{\tilde{X}}\right)=D_{h}\left(\tilde{\mathscr{M}}\left(D_{\tilde{X}}, K\right)\right) .
\end{aligned}
$$

2.16.3. Let us consider again the last example 3. Let $U(\mathfrak{h})$ be the enveloping algebra of the torus $H$. Given $M \in \mathscr{M}\left(U(\bar{k}) \otimes \mathscr{D}_{\tilde{X}}\right)$, it is naturally a $U(h)$ module via $w$ (see 2.2). This action $w$ commutes with the $\left(U(\bar{k}) \otimes D_{\tilde{X}}\right)$-module structure on $M$. Indeed, this follows from (ii) in 2.2, (i) in 2.5 and the fact that the $K^{0}$-action commutes with the $H$-action. Recall that $K$ acts on $U(\mathfrak{h})$ 
via $\tau$. Extend this action to the action of $F=K \ltimes H$, where $H$ acts trivially. Then the $U(\mathfrak{h})$-module structure on $M$ is compatible with the $F$-action via $\tau$ :

$$
a(\xi m)=\tau(a)(\xi) a(m), a \in F, \xi \in U(\mathfrak{h}), m \in M .
$$

Let $I \subset U(\mathfrak{h})$ be a $K$-invariant ideal. Consider the usual full subcategories

$$
\begin{gathered}
\mathscr{M}_{\tilde{I}}\left(U(\bar{k}) \otimes \mathscr{D}_{\tilde{X}}\right) \subset \mathscr{M}\left(U(\bar{k}) \otimes \mathscr{D}_{\tilde{X}}\right), \\
D_{\tilde{I}}^{b}\left(U(\bar{k}) \otimes \mathscr{D}_{\tilde{X}}\right) \subset D^{b}\left(U(\bar{k}) \otimes \mathscr{D}_{\tilde{X}}\right),
\end{gathered}
$$

and the natural functor

$$
\tilde{\nu}^{\prime}: D^{b}\left(\mathscr{M}_{\tilde{I}}\left(U(\bar{k}) \otimes \mathscr{D}_{\tilde{X}}\right)\right) \rightarrow D_{\tilde{I}}^{b}\left(\mathscr{M}\left(U(\bar{k}) \otimes \mathscr{D}_{\tilde{X}}\right)\right) .
$$

This is the functor $\tilde{\nu}$ in 2.5 under the identifications of example 3 above. Theorem 2.5.1 claims that this functor is an equivalence. This follows from the following general theorem.

2.16.4. Let $Y$ be an $F$-variety and $\mathscr{B}_{Y}=\left(B_{Y}, F \mid K, d\right)$ be a Harish-Chandra $D G$-algebra (2.16.1). Let $Z$ be a finitely generated commutative ring with an algebraic action $\tau: F \rightarrow \operatorname{Aut}(Z)$. Assume that the ring $Z$ acts by endomorphisms of the category $\mathscr{M}\left(\mathscr{B}_{Y}\right)$. More precisely, for every $M \in \mathscr{M}\left(\mathscr{B}_{Y}\right)$ there is given a $Z$-module structure, which commutes with the differential on $M$, with the $B_{Y}$-action, and

$$
a(z m)=\tau(a)(z) a(m), \quad z \in Z, a \in F, m \in M .
$$

Moreover assume that this $Z$-module structure is preserved by morphisms in $\mathscr{M}\left(\mathscr{B}_{Y}\right)$. Let $I \subset Z$ be an $F$-invariant ideal and $\mathscr{M}_{I}\left(\mathscr{B}_{Y}\right) \subset \mathscr{M}\left(\mathscr{B}_{Y}\right)$ be the full subcategory of $I$-finite modules. Let $D_{\tilde{I}}^{b}\left(\mathscr{B}_{Y}\right) \subset D^{b}\left(\mathscr{B}_{Y}\right)$ be the full subcategory of $\mathscr{B}_{Y}$-modules with $I$-finite cohomologies. We have the natural functor

$$
\bar{\nu}: D^{b}\left(\mathscr{M}_{\tilde{I}}\left(\mathscr{B}_{Y}\right)\right) \rightarrow D_{\tilde{I}}^{b}\left(\mathscr{B}_{Y}\right)
$$

Theorem. In the above notations assume that the sheaf of algebras $B_{Y}$ is nonpositively graded and is locally left Noetherian. Then the functor $\bar{\nu}$ is an equivalence of categories.

The proof of this theorem is similar to the proof of Theorem 1.16.1 above and we will not repeat it.

\section{A CONJECTURE AND EXAMPLES}

3.1. Let $X$ be a smooth variety acted upon by an affine algebraic group $G$. Consider the $G$-equivariant derived category $D_{G}^{b}\left(D_{X}\right)$ of $D$-modules on $X$ (2.11). Let $\mathscr{M}\left(D_{X}, G\right)$ be the abelian category of $G$-equivariant $D_{X}$-modules. We have a natural fully faithful functor $\mathscr{M}\left(D_{X}, G\right) \rightarrow D_{G}^{b}\left(D_{X}\right)$ which identifies $\mathscr{M}\left(D_{X}, G\right)$ with the full subcategory of complexes concentrated in degree zero. Thus we obtain the functor

$$
\beta: D^{b}\left(\mathscr{M}\left(D_{X}, G\right)\right) \rightarrow D_{G}^{b}\left(D_{X}\right)
$$

This functor is not an equivalence in general (take for example $X=p t, G=$ $\mathbb{C}^{*}$ ). However it seems that if the $G$-action on $X$ is free at a general point of $X$, then $\beta$ should be an equivalence. More precisely, we have the following 
3.2. Conjecture. Suppose that the connected component of the stabilizer of a general point in $X$ is unipotent. Then $\beta$ is an equivalence.

3.3. Remark. The importance of this conjecture is clear. For example, the category $D^{b}\left(\mathscr{M}\left(D_{X}, G\right)\right)$ often has a representation-theoretical meaning (e.g. via the localization). But some of the usual geometric properties fail in this category. For example, let $Y \subset X$ be a closed smooth $G$-stable subvariety. Then the obvious functor

$$
D^{b}\left(\mathscr{M}\left(D_{Y}, G\right)\right) \rightarrow D_{Y}^{b}\left(\mathscr{M}\left(D_{X}, G\right)\right)
$$

is not an equivalence in general: take $X=\mathbb{C}, Y=$ the origin in $\mathbb{C}, G=$ $\mathbb{C}^{*}$ with the natural $\mathbb{C}^{*}$-action on $\mathbb{C}$. (This functor is an equivalence in the nonequivariant situation by Kashiwara's theorem [B].) On the other hand, the equivariant derived category $D_{G}^{b}\left(D_{X}\right)$ has all the usual geometric and functorial properties of the nonequivariant derived category. So it is useful to know that in some cases these two categories are equivalent.

We can easily deduce from our results in section 1 the following theorem related to the above conjecture.

3.4. Theorem. Let $X$ be a smooth $D$-affine variety (e.g. $X$ is affine) acted upon by an affine algebraic group $G$. Let $A=\Gamma\left(X, D_{X}\right)$ be the ring of global differential operators on $X$ and $U(\mathfrak{g})$ be the universal enveloping algebra of $\mathfrak{g}:=$ Lie $G$. Consider the canonical homomorphism

$$
j: U(\mathfrak{g}) \rightarrow A
$$

induced by the G-action on $X$. Assume that $A$ is a projective right $U(\mathfrak{g})$-module via $j$. Then the functor

$$
\beta: D^{b}\left(\mathscr{M}\left(D_{X}, G\right)\right) \rightarrow D_{G}^{b}\left(D_{X}\right)
$$

is an equivalence of categories.

Proof. The functor $\beta$ is a composition of two functors

$$
D^{b}\left(\mathscr{M}\left(D_{X}, G\right)\right) \stackrel{\beta^{\prime}}{\rightarrow} D_{h}^{b}\left(\mathscr{M}\left(D_{X}, G\right)\right) \stackrel{\beta^{\prime \prime}}{\rightarrow} D_{G}^{b}\left(D_{X}\right) .
$$

By Theorem 2.13 the functor $\beta^{\prime \prime}$ is always an equivalence. So it suffice to prove that $\beta^{\prime}$ is such. Since $X$ is $D$-affine, we have " $D_{X}$-modules" = " $A$-modules". Moreover, the $G$-action on $A$ and the homomorphism $j: U(\mathfrak{g}) \rightarrow A$ make $(A, G)$ into a Harish-Chandra pair (1.4), and

$$
\mathscr{M}\left(D_{X}, G\right)=\mathscr{M}(A, G), D^{b}\left(\mathscr{M}\left(D_{X}, G\right)\right)=D^{b}(\mathscr{M}(A, G)) .
$$

Similarly,

$$
D_{h}^{b}\left(\mathscr{M}\left(D_{X}, G\right)\right)=D_{h}^{b}(\mathscr{M}(A, G)) .
$$

Under these identifications the functor $\beta^{\prime}$ is the functor

$$
D^{b}(\mathscr{M}(A, G)) \rightarrow D_{h}^{b}(\mathscr{M}(A, G)),
$$

which is an equivalence by Theorem 1.6. 
3.5. Consider a $(\mathfrak{g}, K)$-pair $(1.1)$, where $\mathfrak{g}$ is semisimple. Let $U(\mathfrak{g})$ be the enveloping algebra of $\mathfrak{g}, Z$ the center of $U(\mathfrak{g})$, and $Z^{+}=\operatorname{Ker} \theta_{0}: Z \rightarrow \mathbb{C}$ the augmentation ideal. Put

$$
U_{0}:=U(\mathfrak{g}) / Z^{+} U(\mathfrak{g})
$$

Let $X$ be the flag variety of $\mathfrak{g}$. We know that $X$ is $D$-affine and that the natural homomorphism $U(\mathfrak{g}) \rightarrow \Gamma\left(X, D_{X}\right)$ induces an isomorphism $U_{0} \stackrel{\sim}{\rightarrow}$ $\Gamma\left(X, D_{X}\right)$. Consider the natural $K$-action on $X$. Let $k=$ Lie $K$. The natural homomorphism $U(k) \rightarrow \Gamma\left(X, D_{X}\right)$ coming from the $K$-action on $X$ factors through the embedding $U(k) \hookrightarrow U(\mathfrak{g})$ given in the $(\mathfrak{g}, K)$-pair. The following theorem is an immediate consequence of Theorem 3.4.

3.5.1. Theorem. Assume that in the above notations the algebra $U_{0}$ is a projective right $U(k)$-module via the embedding $U(k) \hookrightarrow U(\mathfrak{g})$. Then the functor

$$
\beta: D^{b}\left(\mathscr{M}\left(D_{X}, K\right)\right) \rightarrow D_{K}^{b}\left(D_{X}\right)
$$

is an equivalence of categories.

The following theorem was proved in [BL2] (we keep the notations of 3.5).

3.5.2. Theorem. Assume that the stabilizer in $K$ of a general point in $X$ is finite. Then $U_{0}$ is a free $U(k)$-module.

Theorems 3.5.1 and 3.5.2 together provide a special case of Conjecture 3.2.

By the usual localization theorem $(2.9 .1,2.9 .2)$ we know that

$$
\mathscr{M}_{0}(\mathfrak{g}, K) \simeq \mathscr{M}\left(D_{X}, K\right),
$$

where $\mathscr{M}_{0}(\mathfrak{g}, K)$ is the category of $(\mathfrak{g}, K)$-modules with the trivial central character. Combining this result with Theorems 3.5.1, 3.5.2 we obtain the following

3.5.3. Theorem. Consider a (g, K)-pair (1.1), where $\mathfrak{g}$ is a semisimple Lie algebra. Let $\mathscr{M}_{0}(\mathfrak{g}, K)$ be the category of $(\mathfrak{g}, K)$-modules with the trivial central character. Let $X$ be the flag variety for $\mathfrak{g}$. Assume that the stabilizer in $K$ of a general point in $X$ is finite. Then there exists a natural equivalence of categories

$$
D^{b}\left(\mathscr{M}_{0}(\mathfrak{g}, K)\right) \simeq D_{K}^{b}\left(D_{X}\right),
$$

where $D_{K}^{b}\left(D_{X}\right)$ is the $K$-equivariant derived category of D-modules on $X$ (2.11).

3.5.4. Examples. In the above theorem let $G$ be the adjoint group of $\mathfrak{g}$. To satisfy the assumptions of the last theorem one may take for example $K=U$ to be a unipotent subgroup of $G$ or $K=$ the complexification of the maximal compact subgroup of $G$.

3.6. Let $G$ be an affine algebraic group and $K \subset G$ a subgroup. Put $Y=G / K$ with the natural action of $G$.

3.6.1. Proposition. In the above notations the functor

$$
\beta: D^{b}\left(\mathscr{M}\left(D_{Y}, G\right)\right) \rightarrow D_{G}^{b}\left(D_{Y}\right)
$$

(3.1) is an equivalence if and only if the rank of $K$ is zero. 
Proof. Indeed, in this case both categories $\mathscr{M}\left(D_{Y}, G\right)$ and $D_{G}^{b}\left(D_{Y}\right)$ are induced from a point $p t$ with the (trivial) $K$-action ([BL1]). That is,

$$
\begin{gathered}
\mathscr{M}\left(D_{Y}, G\right) \simeq \mathscr{M}\left(D_{p t}, K\right), \\
D_{G}^{b}\left(D_{Y}\right) \simeq D_{K}^{b}(p t) .
\end{gathered}
$$

But $\mathscr{M}\left(D_{p t}, K\right)$ is a semisimple category which is equivalent to the category of $\left(K / K^{0}\right)$-modules $\left(K^{0}\right.$ is the connected component of $\left.K\right)$, and $D_{K}^{b}(p t)$ is semisimple if and only if $K$ has rank zero ([BL1]). This proves the proposition.

The following theorem is an immediate consequence of 3.4, 3.6.1.

3.6.2. Theorem. In the above notations assume that the space $Y=G / K$ is D-affine (e.g. $Y$ is affine). Put $A:=\Gamma\left(Y, D_{Y}\right)$, the ring of global differential operators on $Y$. Let $U(\mathfrak{g})$ be the enveloping algebra of $\mathfrak{g}:=\operatorname{Lie} G$. Consider the canonical homomorphism $j: U(\mathfrak{g}) \rightarrow A$ induced by the G-action on $Y$. Assume that the group $K$ has a positive rank. Then the ring $A$ considered as a right $U(\mathfrak{g})$ - module via $j$ is not a projective module.

3.6.3. Problem. In the notations of the last theorem study the algebra $\operatorname{Ext}_{U(\mathfrak{g})}(A, A)$.

\section{REFERENCES}

[AM] M. F. Atiyah and I. G. MacDonald, Introduction to commutative algebra, Addison-Wesley, Reading, MA, 1969.

[B] A. Borel, Algebraic D-modules, Perspectives in Math., vol. 2, Academic Press, New York and London, 1986.

[BB] A. Beilinson and J. Bernstein, A proof of Jantzen conjectures, Adv. Soviet Math., vol. 16, Amer. Math. Soc., Providence, RI, 1993.

[Be] A. Beilinson, On the derived category of perverse sheaves, Lecture Notes in Math., vol. 1289, Springer-Verlag, Berlin and New York, 1987.

[BG] J. Bernstein and S. Gelfand, Tensor products of finite and infinite dimensional representations of semisimple Lie algebras, Compositio Math. 41 (1980), 245-285.

[BL1] J. Bernstein and V. Lunts, Equivariant sheaves and functors, Lecture Notes in Math., vol. 1578, Springer-Verlag, Berlin and New York, 1994.

[BL2] __ A simple proof of Kostant's theorem that $U(\mathfrak{g})$ is free over its center, preprint.

[DV] M. Duflo and M. Vergne, Sur le foncteur de Zuckerman, C.R. Acad. Sci. Paris Ser. I 304 (1987), 467-469.

[II] L. Illusie, Complex cotangent et deformation, Lecture Notes in Math., vols. 239, 283, Springer-Verlag, Berlin and New York, 1971, 1972.

[Sp] N. Spaltenstein, Resolutions of unbounded complexes, Compositio Math. 65 (1988), 121-154.

School of Mathematical. Sciences, Tel Aviv University, Israel

DEPARTMENT OF MATHEMATICS, INDIANA UNIVERSITY, BLOOMINGTON INDIANA 47405

E-mail address: vlunts@ucs.indiana.edu 